\title{
Complex Spike Activity of Purkinje Cells in the Oculomotor Vermis during Behavioral Adaptation of Monkey Saccades
}

\author{
Robijanto Soetedjo ${ }^{2,3}$ and Albert F. Fuchs $s^{1,3}$ \\ Departments of ${ }^{1}$ Physiology and Biophysics, ${ }^{2}$ Biological Structure, and ${ }^{3}$ Washington Regional Primate Research Center, University of Washington, Seattle, \\ Washington 98195-7330
}

\begin{abstract}
Throughout life, the oculomotor system can correct itself when saccadic eye movements become inaccurate. This adaptation mechanism can be engaged in the laboratory by displacing the target when the saccade toward it is in flight. Forward and backward target displacements cause gradual increases and decreases in saccade amplitude, respectively. Equipped with this paradigm, we asked whether Purkinje cells (P-cells) in the vermis of the oculomotor cerebellum, lobules VIc and VII, changed their complex spike (CS) discharge during the behavioral adaptation of horizontal saccades. We tested the hypothesis that CS activity would change only when a targeting saccade caused an error in eye position relative to the target, i.e., during the error interval between the primary and corrective saccades. We examined only those P-cells whose simple spike activity exhibited either a burst or pause with saccades in several directions. Approximately $80 \%$ of such P-cells exhibited an increase in CS activity during the error interval when the adaptation paradigm imposed horizontal eye-position errors in one direction and a decrease in activity for errors in the other. As adaptation progressed and errors were reduced, there was no consistent change in the CS activity. These data suggest that the CS activity of P-cells in the oculomotor vermis signals the direction but not the magnitude of eye-position error during saccade adaptation. Our results are consistent with cerebellar learning models that have been proposed to explain adaptation of the vestibulo-ocular reflex so similar mechanisms may also underlie plasticity of this precision voluntary oculomotor behavior.
\end{abstract}

Key words: saccades; cerebellum; complex spikes; adaptation; motor learning; climbing fiber

\section{Introduction}

Saccadic eye movements redirect the line of sight rapidly and accurately from one interesting object to another. Growth, aging, and injury continually challenge the saccadic system with changing demands. Nevertheless, saccades remain accurate until we are well into our 70s (Warabi et al., 1984; Munoz et al., 1998). Therefore, the saccadic system is capable of adapting its motor behavior. Where and how does this adaptation occur?

Saccade commands originate in a variety of cortical structures and descend to the brainstem, where many of these signals converge on the superior colliculus (SC). The SC influences the motoneurons that produce saccades through two pathways (for review, see Scudder et al., 2002). The first is a relatively direct pathway to a premotoneuronal circuit [burst generator (BG)] (see Fig. 1), which is configured to discharge a burst of spikes for saccades. The second is an indirect pathway that reaches the same BG through the oculomotor cerebellum. Signals from the SC are

Received 0ct. 31, 2005; revised June 13, 2006; accepted June 14, 2006.

This work was supported by National Institutes of Health Grants EY00745 and RR00166 (A.F.F.) and F32EY015046-02 (R.S.). We appreciate the technical support of Monica Ibarreta and Bob Cent and the valuable comments of C. R. S. Kaneko, L. Ling, and Avery Weiss on a previous version of this manuscript. Finally, we are very grateful to Steve Lisberger and Steve Highstein for their careful reading of this manuscript and their insightful suggestions.

Correspondence should be addressed to Dr. Albert F. Fuchs, 1959 NE Pacific Street, HSB 1421, Washington Regional Primate Research Center, Box 357330, University of Washington, Seattle, WA 98195-7330. E-mail: fuchs@u.washington.edu.

D0I:10.1523/JNEUROSCI.4658-05.2006

Copyright $\odot 2006$ Society for Neuroscience $\quad$ 0270-6474/06/267741-15\$15.00/0 relayed through a pontine nucleus (nucleus reticularis tegmenti pontis) to the oculomotor vermis (OMV), whose Purkinje cells (P-cells) inhibit neurons in the caudal fastigial nucleus (CFN). Because the timing of the burst of CFN neurons varies with saccade size (Ohtsuka and Noda, 1991), it is thought to shape the duration of the BG burst, thereby controlling saccade amplitude (Fuchs et al., 1993).

This cerebellar pathway not only helps ensure that individual saccades are accurate but also appears to be involved with saccade adaptation. Damage of both the OMV and CFN destroyed a monkey's ability to correct saccades made inaccurate by impairing the eye muscles (Optican and Robinson, 1980). Also, muscimol inactivation of the CFN eliminated saccade adaptation to eye-position errors that occur when saccades are made inaccurate by behavioral manipulations (Robinson et al., 2002). However, after the effects of the muscimol had dissipated in these experiments, adaptation was revealed, indicating it had indeed occurred at an upstream site but could not be expressed through the compromised CFN. A likely upstream site is the OMV in which lesions abolished rapid saccade adaptation (Barash et al., 1999).

A model of motor adaptation in the vermis has been suggested by Marr (1969) and Albus (1971). P-cells in the vermis receive mossy fiber and climbing fiber inputs (see Fig. 1) that produce frequent simple (SSs) and infrequent complex (CSs) spikes, respectively (inset). When there is an error in motor performance, the model hypothesized that CS activity would change and affect the discharge of the simple spikes, which, in turn, would modify 
brainstem activity to adjust a motor response and reduce the error. This model has had some success in accounting for the adaptation that occurs in another oculomotor behavior, the vestibulo-ocular reflex (Ito, 2005).

Here we test whether this model could apply to the saccadic system by recording CS activity while a monkey underwent the gradual changes in saccade amplitude produced by displacing the target as the eyes moved toward it (McLaughlin, 1967; Straube et al., 1997).

Parts of this work have been published previously in abstract form (Soetedjo and Fuchs, 2003).

\section{Materials and Methods}

General procedures

The techniques that we use to monitor eye movement, to train monkeys to follow small jumping targets with their eyes, and to record extracellular action potentials have been described in detail previously (Soetedjo et al., 2002). Briefly, eye movements were measured with an electromagnetic technique that was approximately linear within $\pm 20^{\circ}$ horizontally and vertically and had a sensitivity of $15 \mathrm{~min}$ arc (Fuchs and Robinson, 1966). The animals were rewarded with applesauce when they kept their eyes within $2^{\circ}$ of the horizontal and vertical location of a jumping target spot for $\sim 2-3 \mathrm{~s}$ no matter where it moved. A jumping target was produced by either the sequential illumination of stationary light emitting diodes subtending $0.25^{\circ}$ of arc and spaced at $1^{\circ}$ intervals or projecting a laser spot off two orthogonal galvanometer-controlled mirrors to a tangent screen facing the monkey. Finally, unit activity was recorded extracellularly through homemade tungsten microelectrodes that were driven to the cerebellum by means of either a hydraulic microdrive or a stepper motor.

All of our procedures complied with the guidelines imposed by the Institutional Animal Care and Use Committee of the University of Washington. Surgical and experimental protocols were conducted in accordance with the National Institutes of Health Guide for the Care and Use of Laboratory Animals and in compliance with the recommendations from the Institute of Laboratory Animal Resources and the International Association for Assessment and Accreditation of Laboratory Animal Care.

\section{Identification of units}

In these experiments, we recorded neuronal activity from the OMV (lobules VIc and VII) while a monkey was undergoing behaviorally induced adaptation of saccade amplitude. Before adaptation, we identified units in the OMV by requiring the monkeys to make $15^{\circ}$ saccades from straight ahead in eight different directions [0 (rightward), 45, 90 (upward), 135, 180 (leftward), 225, 270 (downward), and $315^{\circ}$ ]. Under these conditions, we easily could identify the vermal lobules in which the background activity exhibited a phasic change in firing with saccades, usually in all directions. We then isolated saccade-related units with traditional action potentials, i.e., SSs, and required that they discharge a CS to be considered as P-cells. We considered that the CS and SSs were recorded from the same cell if the occurrence of the CS was associated with a subsequent pause in SS activity. Figure 1 shows a representative extracellular P-cell recording (inset), although the separation of SSs (gray) and CSs (black) was often not this clear (see below). The presence of a CS was very important because we also isolated other units that had a vigorous discharge with saccades, usually in limited directions, had no CS, and were likely the axons of afferents from neurons in the brainstem. In the current experiments, we did not examine those P-cells that did not exhibit phasic changes in SS activity related to saccades, even if they were located in a folium with phasic background activity.

\section{Induction of saccade adaptation}

We induced saccade amplitude increases and decreases with the McLaughlin (1967) paradigm. In this paradigm, a saccade to a target step is detected and the target is made to jump forward or backward, respectively, during the saccade when vision is poor. When the saccade lands, it appears to have either fallen short or overshot the target, respectively. After many repetitions of this deception, saccade amplitude gradually
Cerebellum

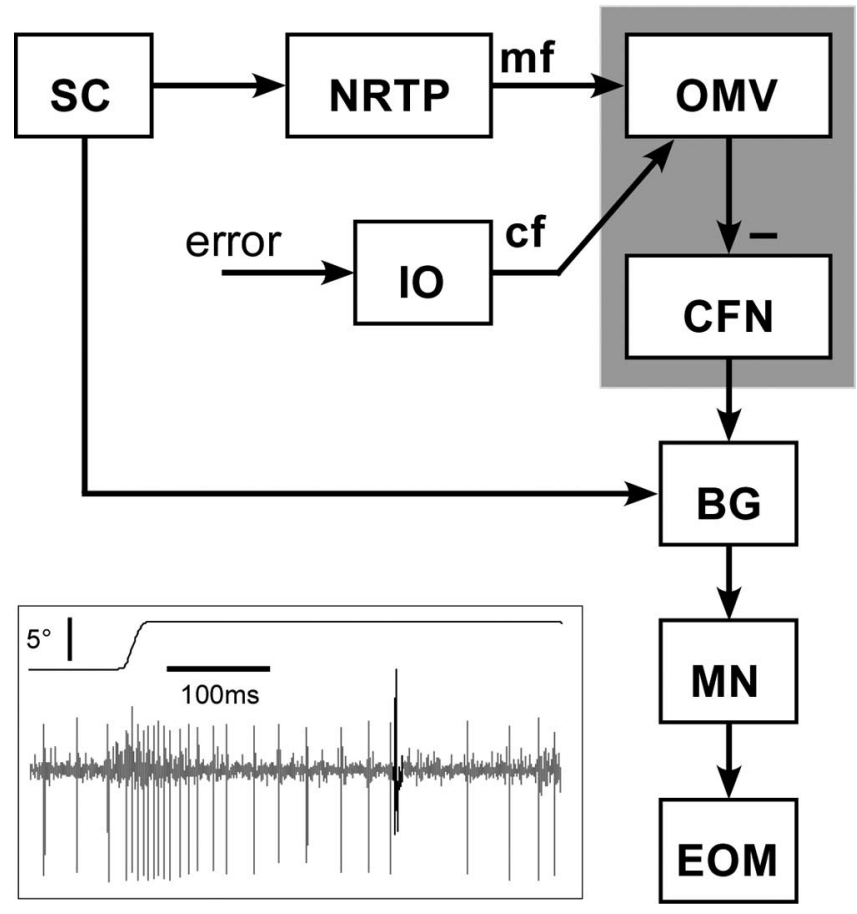

Figure 1. Highly schematic model of the brainstem and cerebellar components of the saccadic system. Signals from the $\mathrm{SC}$ reach the brainstem BG for horizontal saccades through a relatively direct pathway and also via a side loop through the oculomotor cerebellum (shaded), which consists of the OMV (lobules VIc and VII) and the CFN to which it projects. In addition to a mossy fiber ( $\mathrm{mf}$ ) input from the $\mathrm{SC}$ delivered via the nucleus reticularis tegmenti pontis (NRTP), the $0 M V$ receives climbing fiber ( $\mathrm{Cf}$ ) signals exclusively from the inferior olive (I0), which has been proposed to relay an error signal. The mossy fiber input results in the high rate simple spike discharge (inset, gray), whereas the climbing fiber input produces the complex spike (black), which occurs much less frequently. The P-cell in the inset shows a burst of simple spikes for the $5^{\circ}$ saccade shown in the top trace. MN, Horizontal motoneurons, EOM; extraocular muscles.

changes to bring the eye more nearly to the displaced target location. If saccades to target steps of one amplitude and direction experience an intrasaccadic target adapt jump, $\sim 500-1000$ erroneous trials are required for nearly complete adaptation in the monkey (Straube et al., 1997; Kojima et al., 2004). Because the number of trials required for nearly complete adaptation is directly proportional to the number of adapted target directions, it was impractical to adapt target steps in eight different directions. Because all 27 of our neurons had simple spike activity that showed either a burst or pause for saccades in all directions, we decided to adapt horizontal saccades only. This strategy proved to be not only practical but also productive because 21 of our $27 \mathrm{P}$-cells responded to horizontal errors to either the right or left.

In all experimental sessions, we adapted saccades to $15^{\circ}$ horizontal target steps that started from pseudorandom initial horizontal eye positions. A behavioral control program required the monkeys to fixate within $\pm 2^{\circ}$ of the target for at least $0.6 \mathrm{~s}$. A new $15^{\circ}$ horizontal target jump was initiated every $1-1.3 \mathrm{~s}$. This fast pace of saccades was necessary to collect the maximum amount of data before we lost the neuron. We used $5^{\circ}$ intrasaccadic adapt jumps. For each adaptation block, the adapt jump was forward for saccades in one direction and backward for saccades in the other so that both the amplitude and direction of the saccadic error within a block was the same. Because backward adapt jumps returned the target toward the center, there were always more trials with backward than forward adapt jumps. Our choice of the error direction in the first block of trials was random. Occasionally, when the CS could easily be identified on-line by visual inspection of the ongoing unit activity, we checked the efficacy of the chosen error direction by monitoring CS activity. If there was no discernible increase in CS discharge for the error direction we had chosen, we often reversed the adaptation conditions 
A

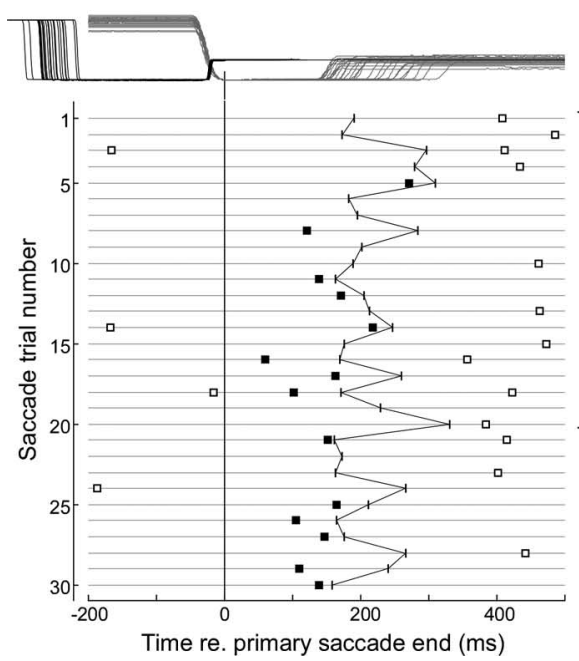

B

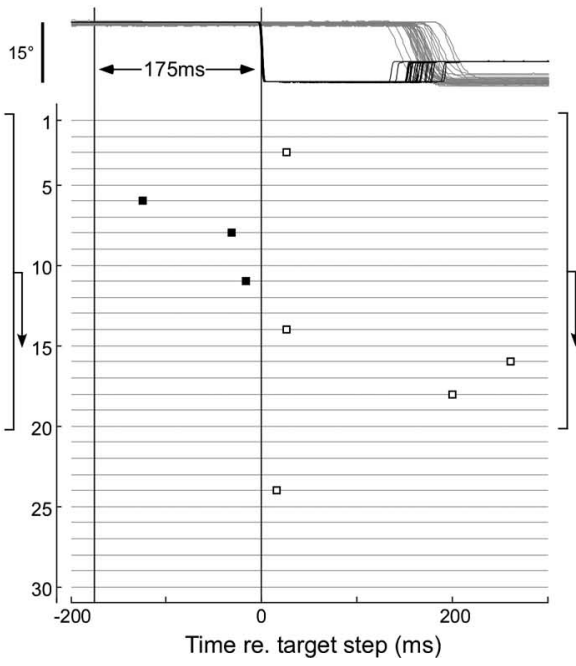

Figure 2. Method of complex spike analysis illustrated on the first 30 trials of a gain reduction block. $A$, Calculation of the first term in Equation 1 (see Materials and Methods). Top panel shows all 30 saccadic responses aligned on the end of the primary saccade, and the bottom panel shows their associated CSs (squares). All of the CSs (filled squares) that occur in the interval from the end of the primary saccade to the onset of the corrective saccade plus $25 \mathrm{~ms}$ (vertical tics, jagged line) in the first 20 trials are added, and this sum is divided by the sum of all of the intersaccadic intervals in those trials to determine the average firing rate. $\boldsymbol{B}$, Calculation of the second term in Equation 1. For the same 20 trials, CSs (filled squares) in the interval $175 \mathrm{~ms}$ before the target steps are counted and divided by $20 \times 0.175 \mathrm{~s}$ to determine average background rate. The number generated in $\boldsymbol{B}$ is subtracted from that in $\boldsymbol{A}$ to determine $\Delta \mathrm{CS}_{1}$. The process in $\boldsymbol{A}$ and $\boldsymbol{B}$ is then shifted to start at saccade 2 and repeated to determine $\Delta \mathrm{CS}_{2}$, etc., thereby producing a 20 -saccade running average of the $C S$ discharge rate above the baseline. Open squares identify $C S s$ not used for analysis.

after 200-300 trials of both leftward and rightward saccades before adaptation had gone to completion.

\section{Specific conditions during a typical recording}

In each experiment, we attempted to collect several blocks of data. First, we determined whether the SS activity of the P-cell had a preferred direction by requiring the monkey to make saccades to $15^{\circ}$ target steps in eight directions every $45^{\circ}$ from 0 (rightward) to $315^{\circ}$. We collected $\sim 20-25$ saccades for each direction. Second, we collected $15^{\circ}$ horizontal saccades (50-100 saccades each to the left and right); in 10 experiments, we collected $5^{\circ}$ horizontal saccades as well. Third, we subjected the monkey to one or more adaptation paradigms. While recording from 25 P-cells, we elicited forward adaptation in one direction and backward adaptation in the other. For 14 of these, we held the P-cell long enough to reverse the direction of the adaptation at least once. In two experiments early in the project, we used backward adaptation steps for both leftward and rightward saccades.

During the experiment, a custom computer program running in Spike2 (Cambridge Electronic Design, Cambridge, UK) controlled the data acquisition box (Power 1401) and analyzed the incoming data online. The unit signal was digitized at $50 \mathrm{kHz}$, and the eye and target position signals were sampled at $1 \mathrm{kHz}$. The program displayed histograms of SS activity for $15^{\circ}$ saccades in eight different directions. The histogram display is similar to the one shown in Figure 3. The program also plotted the gain (eye movement amplitude/target amplitude) of the saccades so we could monitor the progress of the adaptation. Occasionally, we attempted to trigger on CSs to monitor their frequency of occurrence during the time of the position error between the primary and corrective saccades. Once an experiment ended, the data were saved to a disk for off-line analysis.

\section{Data analysis}

General data processing. We performed off-line analyses of CS activity with custom programs running in Spike2 and Matlab (MathWorks, Natick, MA) software. Only a few neurons had CSs that were sufficiently different from the SSs that they could be recognized electronically (e.g., the unit in Fig. 1). However, CSs in all other P-cells could easily be recognized by eye when epochs of single-unit activity were scrolled on a video monitor. A.F.F. scored each CS manually on the basis of the following criteria: the CS always was positive followed by several smaller wavelets, always followed by a pause in SS discharge, and often followed the preceding SS by $<2 \mathrm{~ms}$. The identification of CSs was done on records without the associated eye position and target traces to eliminate any bias in the scoring. Once identified, the CS was converted to a spike event that was placed on the associated eye and target data.

Each saccade trial was considered separately. A saccade analysis program written in Spike2 first calculated the velocities of the horizontal and vertical components of eye and target position. To identify a trial, the program determined when the velocity of the target step exceeded $300 \%$ and then searched for a primary saccade that occurred 50-500 ms later. The saccade was detected when the sum of the rectified horizontal and vertical eye velocities exceeded $100 \%$ s. Then, the beginning and end of each component of the saccade were marked as the times when saccade velocity first exceeded and then next fell below $20 \%$ s. Once the primary saccade was identified, the program used the same criteria to search for the corrective saccade within $500 \mathrm{~ms}$ from the end of the primary saccade. The saccade analysis program determined the attributes of each saccade, e.g., latency, onset, offset, amplitude, duration, peak velocity, etc., and saved both the digitized traces (CS activity and eye and target position and velocity) and the saccade attributes for every trial.

Subsequent analysis was done using custom programs in Matlab. Because occasional saccades were either slow or followed by a long glissade, we included in our analysis only those saccades with durations $\leq$ mean \pm 2 SDs. Because we needed to calculate the baseline CS activity when the monkey was fixating before the initial target step, we further filtered the data with criteria that required both horizontal and vertical eye positions to lie within $\pm 1.5^{\circ}$ of the target before the target stepped. These two restrictions eliminated $<9 \%$ of the saccades.

We tested the correlation between error and CS using both a firing rate and information theory analysis.

Firing rate analysis. Figure 2 shows data from an adaptation experiment to illustrate the firing rate analysis. In Figure $2 \mathrm{~A}$, each of the 30 saccade trials in this segment are superimposed in the top panel and aligned on the end of the primary saccade. The lines from 1 to 30 in the bottom panel show the occurrence of the associated CSs (squares) for each trial. On some trials, e.g., 6 and 7, no CSs occurred at all. Therefore, to get a CS firing rate, we did a running average over 20 trials (vertical brackets with downward arrows) throughout the adaptation block. We started with trials $1-20$ and placed the first average at the middle trial, i.e., 11. Then we considered trials $2-21$ and so forth.

We determined the average CS rate in two intervals during a trial. In Results, we will show that a change in CS rate occurred in the "intersaccadic interval" between the end of primary saccade (vertical line at 0 ) and the beginning of the subsequent corrective saccades, which is indicated by the jagged line. During this interval, there was an eye-position error; therefore, we call this interval the "error interval." Because we observed that the increase in CSs usually did not return to baseline until $25 \mathrm{~ms}$ after the secondary saccade (see Fig. 9), we added $25 \mathrm{~ms}$ to the intersaccadic interval for the calculation of average firing rate during the error interval. In those few trials in which the monkey made no corrective saccade, we used a fixed $175 \mathrm{~ms}$ interval, which was chosen to fit the average mode of intersaccadic intervals from all adaptation trials in 10 random experiments (five from each monkey). The mean of the modes was $173.07 \pm$ $25.97 \mathrm{~ms}$. To determine the CS rate during the intersaccadic interval of 
the $m$ th trial, we performed a 20-trial running average in which we counted all of the spikes (Fig. $2 \mathrm{~A}$, filled squares) in those trials and divided by the sum of the intersaccadic error intervals (first term in Eq. 1). We also determined the baseline CS rate associated with the same trials by counting all of the CSs (Fig. $2 B$, filled squares) that occurred within the $175 \mathrm{~ms}$ interval before the initial target step. This baseline rate was then subtracted in Equation 1 to produce the change in CS rate in the error interval $\left(\Delta \mathrm{CS}_{m}\right)$ :

$$
\Delta \mathrm{CS}_{m}=\frac{\sum_{n=m}^{m+19} \mathrm{CS}_{n}}{\sum_{n=m}^{m+19} \text { Error Interval }_{n}}-\frac{\sum_{n=m}^{m+19} \text { CSbase }_{n}}{0.175 \times 20} .
$$

We also calculated a running average of the associated eye-position error in the intersaccadic interval over the same 20 trials (Error in Eq. 2). Error was taken as the average of [eye position - target position] over the interval from 45 to $53 \mathrm{~ms}$ (eight values every $1 \mathrm{~ms}$ ) after the primary saccade. This delay was chosen to minimize the influence of the changing eye position produced by any postsaccadic glissades (Bahill et al., 1975):

$$
\text { Error }_{m}=\frac{1}{20} \times \sum_{n=m}^{m+19} \text { EyeError }_{n},
$$

where $m=1 \ldots$ [total number of trials -19 ].

To determine whether a unit responded better to a particular error direction, to compare rate changes associated with $5^{\circ}$ primary and corrective saccades, and to compare rate changes during forward and backward adaptations, we used Equation 3. In this calculation, we obtained the average change of rate during the error interval for all of the trials in an entire block of data $\left(\Delta \mathrm{CS}_{\text {block }}\right)$, e.g., a forward adaptation block, by summing all of the spikes and dividing by the sum of the durations of all of the error intervals and then subtracting the average baseline activity:

$\Delta \mathrm{CS}_{\text {block }}=\frac{\sum_{n=1}^{\text {total number of trials }} \mathrm{CS}_{n}}{\sum_{n=1}^{\text {total number of trials }} \text { ErrorInterval }_{n}}$

$-\frac{\sum_{n=1}^{\text {total number of trials }} \text { CSbase }_{n}}{0.175 \times \text { total number of trials }}$,

where $n$ is the sequential trial number, and total number of trials refers to either those within one block of data or those within certain limits of amplitude or error size.

The single value of $\Delta \mathrm{CS}_{\text {block }}$ obtained from Equation 3 does not allow a determination of its variability. To estimate the SD of $\Delta \mathrm{CS}_{\text {block, }}$, we resorted to a statistical bootstrapping technique (Efron and Tibshirani, 1993). This method picks random samples with replacement from a dataset to create bootstrap datasets. The number of data points in a single bootstrap dataset is equal to the total number of trials in a block and produces a single value of $\Delta \mathrm{CS}_{\text {block }}$. The more bootstrap datasets that are generated, the more accurate is the estimation of the distribution of the data. We generated 1000 bootstrap datasets to estimate the mean and SD of $\Delta \mathrm{CS}_{\text {block}}$.

Information theory analysis. We used information theory to determine the probability with which the occurrence of a CS was associated with either an error of a particular size or direction. The conditional probability that the occurrence of a CS was associated with different errors, $i$, was calculated as follows:

$$
p\left(\text { Error }_{i} \mid \mathrm{CS}_{j}\right)=p\left(\mathrm{CS}_{j} \mid \text { Error }_{i}\right) p\left(\text { Error }_{i}\right) / p\left(\mathrm{CS}_{j}\right),
$$

where $j=0$ if there was no CS within the error interval and $j=1$ if there was at least one CS; for those trials that had multiple CSs in the error interval ( $<3 \%$ of all trials), we set $j=1 . i$ indicates a particular error size or error direction condition.

The uncertainty of the overall error was measured as the entropy of the error both when a CS did occur during the error interval of a particular trial and when it did not. This conditional entropy was calculated by the following equation:

$$
H\left(\text { Error } \mid \mathrm{CS}_{j}\right)=-\sum_{i} p\left(\text { Error }_{i} \mid \mathrm{CS}_{j}\right) \log _{2} p\left(\text { Error }_{i} \mid \mathrm{CS}_{j}\right) .
$$

We analyzed the error amplitude data in blocks, which were centered on every degree between 1 and $7^{\circ}$ and had a bin width of $\pm 0.5^{\circ}$. We considered only data in those bins that contained $\geq 50$ trials. Consequently, the $i$ in Equation 5 ranged from 2 to 5, which corresponded to 1-2.32 bits of maximum error entropy.

To test the relationship of CS firing probability to error direction, we grouped the trials according to the sign of the error (left or right). In this calculation, there are only two conditions, i.e., left and right, for the $i$ in Equation 5, so the maximum error entropy is 1 bit.

If a CS occurred preferentially for a certain error condition, the entropy of the error would be less in the trials that a CS occurred. Conversely, if CS occurrence were not related to that error condition, then the entropy would be equal for the trials in which a CS did and did not occur.

Relationship of CS activity with saccade metrics. CS firing exhibited a pause associated with control saccades and the primary saccades of adaptation trials. To determine the relationship of the timing of the pause with the onset and end of the saccade and its duration, we replaced each CS with a Gaussian function with a SD of $10 \mathrm{~ms}$ and summed these smoothed firing rate representations across all of the trials to produce an average spike density function. To identify the pause from this function, we first determined the mean \pm SD rate of the average spike density function over the interval from 250 to $150 \mathrm{~ms}$ preceding the onset of the primary saccade. With the spike density function aligned on the onset of the primary saccade, we took pause onset as the time when the rate decreased by 1 SD from the mean and took pause end as the time when the rate climbed to $1 \mathrm{SD}$ below the mean. To measure the relative timing of pause end and the end of the saccade, we aligned the spike density function on saccade end and determined when the rate climbed to $1 \mathrm{SD}$ below the mean.

\section{Histology}

At the end of the experiment, the first monkey was deeply anesthetized with barbiturate and perfused with physiological saline, followed by $10 \%$ Formalin. The head and brain were immersed in 10\% Formalin. The cerebellum and brainstem were blocked in the stereotaxic sagittal plane and sunk in formol sucrose. The blocked brain was embedded in egg yolk to keep the cerebellar folia from coming apart (Simmons, 1979). The block was cut in frozen section. The sections ( $50 \mu \mathrm{m}$ thick) were stained for Nissl substance and mounted.

\section{Results}

\section{Saccade-related Purkinje cells}

We recorded from 27 saccade-related P-cells in the oculomotor vermis of two monkeys as we produced behavioral adaptation of saccade amplitude. While recording from 14 neurons, we adapted horizontal saccades with both leftward and rightward errors. While recording from 11 other neurons, we adapted horizontal saccades with either leftward or rightward errors. While recording from two neurons early in our experiments, we adapted saccades with a rightward error in one direction and a leftward error in the other, i.e., both backward.

We did adaptation experiments on only those P-cells that exhibited a phasic change in SS activity and ignored those that did not. The changes were either predominantly a burst of spikes (Fig. 3A) or a pause in firing (Fig. 3B). The burst or pause usually occurred for saccades in all directions although not always with 


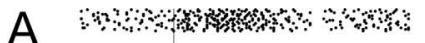
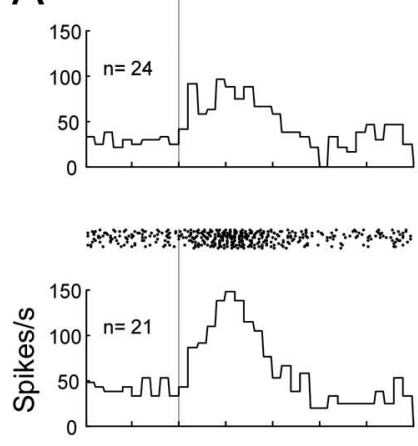

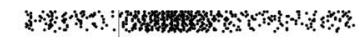

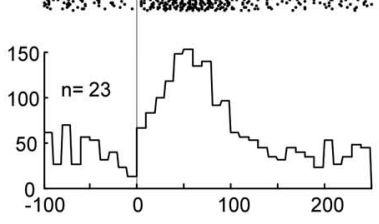

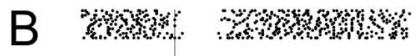
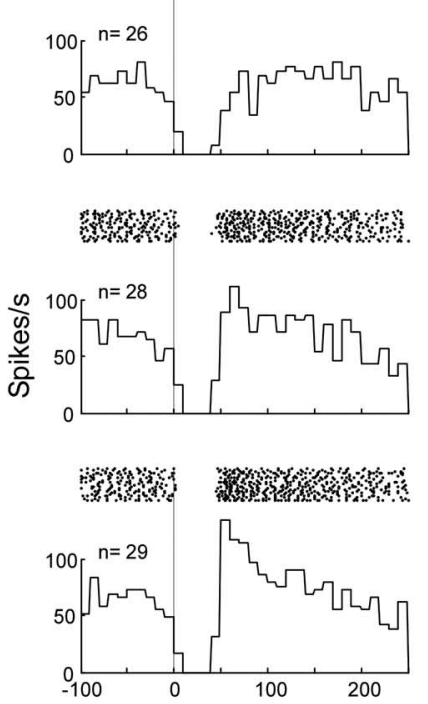
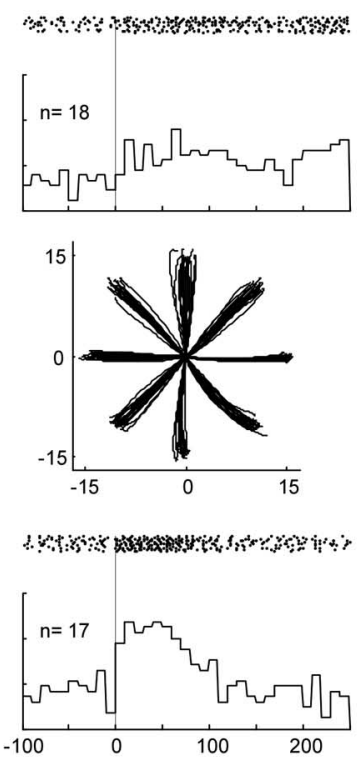

Time re. saccade onset (ms)
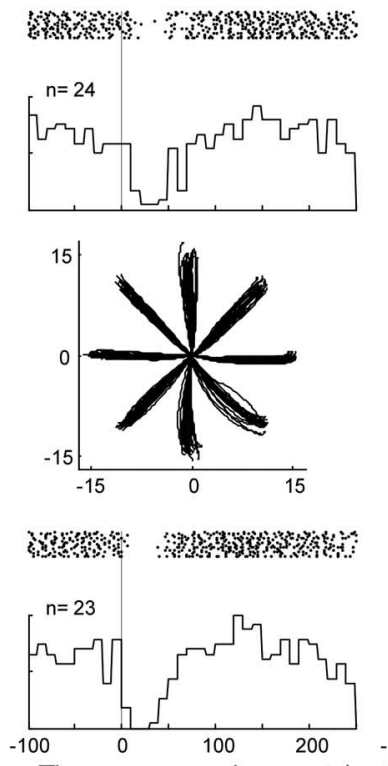

Time re. saccade onset (ms)
4.

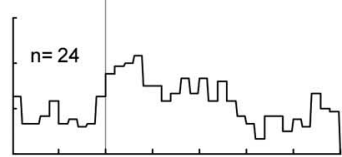

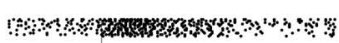

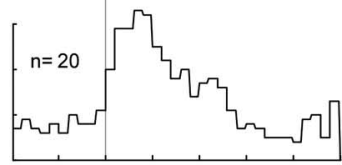

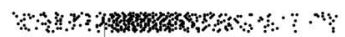

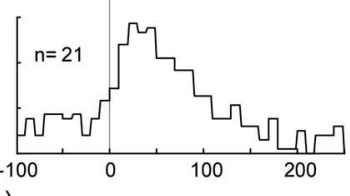

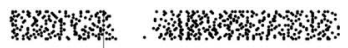
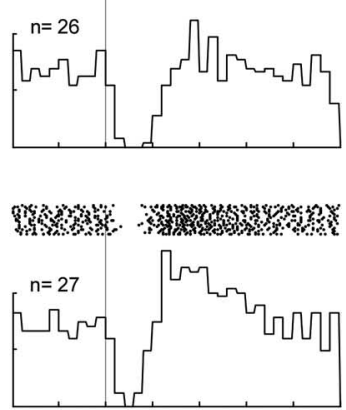

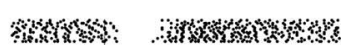

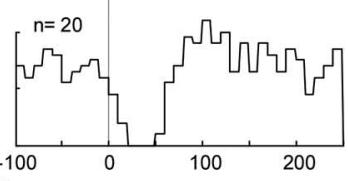

Figure 3. SS discharge of two representative P-cells used in our analyses. All of the P-cells we subjected to CS analysis discharged either a burst $(\boldsymbol{A})$ or pause $(\boldsymbol{B})$ of simple spikes or both associated with saccades, usually in all directions. In both $\boldsymbol{A}$ and $\boldsymbol{B}$, saccades were made to $15^{\circ}$ target steps in eight radial directions from straight ahead and spaced every $45^{\circ}$, as shown in the two middle panels. In the surrounding panels, rasters aligned on saccade onset (dotted lines) show the time of occurrence of each SS of each trial and the associated average histogram ( $10 \mathrm{~ms} / \mathrm{bin}$ ) of all of the trials $(n \geq 17)$ in each direction.

equal intensity. Some neurons exhibited a combination of a burst and pause (Fig. $3 B$, saccades to the left and down). However, for descriptive purposes, we categorized our P-cells as either pausing $(n=8)$ or bursting $(n=19)$ based on their largest change in activity in most directions. The CS behavior of these P-cells was not correlated with their SS discharge patterns.

\section{General findings on CS firing during adaptation}

Figure 4 shows data chosen from an experiment that illustrates all of the salient features of CS activity associated with saccade amplitude adaptation. These include an increase after the primary saccades and a pause preceding the primary saccades (clearest for rightward saccades). We will first consider the increase in CS activity after the primary saccades and in a later section (see below, CS pauses during primary saccades) will examine the pauses.

In this example, we succeeded in adapting saccades to leftward (Fig. $4 A$ ) and rightward (Fig. $4 B$ ) $15^{\circ}$ target steps with both forward and backward $5^{\circ}$ intrasaccadic adapt jumps. In the middle panels, dots indicate the time of occurrence of every CS during the 617 leftward and the 674 rightward adaptation trials arranged sequentially from top to bottom for each direction. During preadaptation trials ( $C, 0$ to $\sim 120$ in each column), a target step elicited a saccade and the target remained in place during the targeting saccade (sample saccade responses shown in black in the top panels). At approximately trial 120 , adaptation trials began. A leftward saccade (column $A$, red trace in top panel) triggered the target to jump forward by $5^{\circ}$ (associated unit data identified by red $\uparrow$ ). This jump caused a leftward error (because it required a leftward corrective saccade to correct it), which induced a gradual increase in saccade amplitude (a sample saccade shown in column $A$, red in top trace) until trial 425 . During pseudorandomly interleaved trials in the opposite direction, a rightward saccade (column $B$, red trace in top panel) caused the target to jump backward by $5^{\circ}$ (associated unit data identified by red $\downarrow$ ). This jump also produced a leftward error, which induced a gradual amplitude decrease (a sample saccade shown in column $B$, red in top panel) until trial 584 . Recall that there were more trials with backward steps because forward steps tended to drive the target to the limits of our display. Through rightward trials 585-674 and leftward trials 426-617, the direction of the adapt jump was reversed so leftward saccades caused a backward target step (blue $\downarrow$ ) and a rightward targeting saccade caused a forward target step (blue $\uparrow)$. During these two adaptation blocks, the error was rightward (see sample adapted saccades in top panels). Note that experiments that involved at least one reversal of the direction of the error were more difficult to complete because they required between 1000 and 2000 total saccade trials.

There was a clear change in the occurrence of CSs during some adaptation conditions. First, there was substantial increase in CS activity on those trials in which the error was leftward (either $\uparrow$ in column $A$ or $\downarrow$ in column $B$, red dots). Furthermore, the increase occurred almost solely during the intersaccadic interval, the time when an eye-position error was present. All of the data in both columns are aligned on the onset of the intersaccadic interval, i.e., the end of the primary saccade. The gray bars indicate the mean and SD of the time that corrective saccades occurred within each adaptation block and hence show the average end of the 
intersaccadic intervals. It is notable that, when the error led to backward adaptation ( $\downarrow$ in column $B$ ), it caused a greater and more sustained increase in CS activity than when the same error led to forward adaptation ( $\uparrow$ in column $A$ ). Second, there was a decrease in overall activity (blue dots) during the error interval on those trials in which the error was rightward (blue arrows), for both leftward (column $A$ ) and rightward (column $B$ ) target steps.

These two changes can be appreciated in the bottom two panels in each column, in which we plot the average CS rate every $25 \mathrm{~ms}$ (forward and backward from time $=0$, the end of the primary saccade) taken from all of the trials during the preadaptation block (black dots), the two leftward error blocks (red dots), and the two rightward error blocks (blue dots). The histograms clearly show that the average CS activity during the intersaccadic interval was highest for leftward errors (red histograms, thick lines). For this and all of our other neurons, we designate the error direction that caused an increase of CS rate above baseline during the error interval as the on-direction error. In addition, this unit showed a decrease in the average CS rate below baseline for rightward errors (blue histograms). We designate this direction as the off-direction error. In the remainder of Results, we use the difference between the CS rate within the error interval and that during baseline conditions on the same trial block $\left(\Delta \mathrm{CS}_{\text {block }}\right)$ (see Materials and Methods) to quantify the change in CS activity during adaptation.

To determine the on-direction error for all of our units, we considered separately the change in CS rate during forward and backward adaptation blocks in each experiment. For the unit in Figure 4 , the $\Delta \mathrm{CS}_{\text {block }}$ in the error interval for the forward ( $\uparrow$ ) and backward $(\downarrow)$ adaptation blocks of leftward saccades was $0.56 \pm 0.22$ and $-1.61 \pm 0.22 \mathrm{spikes} / \mathrm{s}$, respectively. Not only did leftward errors produce a positive $\Delta \mathrm{CS}_{\text {block }}$ (forward adaptation block) but rightward errors produced a negative $\Delta \mathrm{CS}_{\text {block }}$ (backward adaptation block). For the two adaptation blocks of rightward saccades, the $\Delta \mathrm{CS}_{\text {block }}$ was $1.45 \pm 0.16$ and $-0.80 \pm 0.32$ for backward ( $\downarrow$ ) and forward ( $\uparrow$ ) adaptation, respectively. Again, leftward errors (backward block) and rightward errors (forward block) produced changes in CS rate with opposite signs. Therefore, these quantitative data support our previous qualitative observation that both the on- and off-direction errors can be identified clearly for this P-cell.

Figure 5 shows the $\Delta \mathrm{CS}_{\text {block }}$ for each adaptation block for all 27 neurons. For each P-cell, histograms to the left and right of the dashed lines were obtained from trials with leftward and rightward primary saccades, respectively. Red and blue bars identify data obtained with leftward and rightward errors, respectively. In 14 of 27 experiments (units 1-14), we held the neuron long enough to test both rightward and leftward error. For units 1-10, we were able to test one of the error directions a second time. For 10 of these 14 neurons $(1,3-6,9,10,12-14), \Delta \mathrm{CS}_{\text {block }}$ was positive for one error direction and negative for the other for both

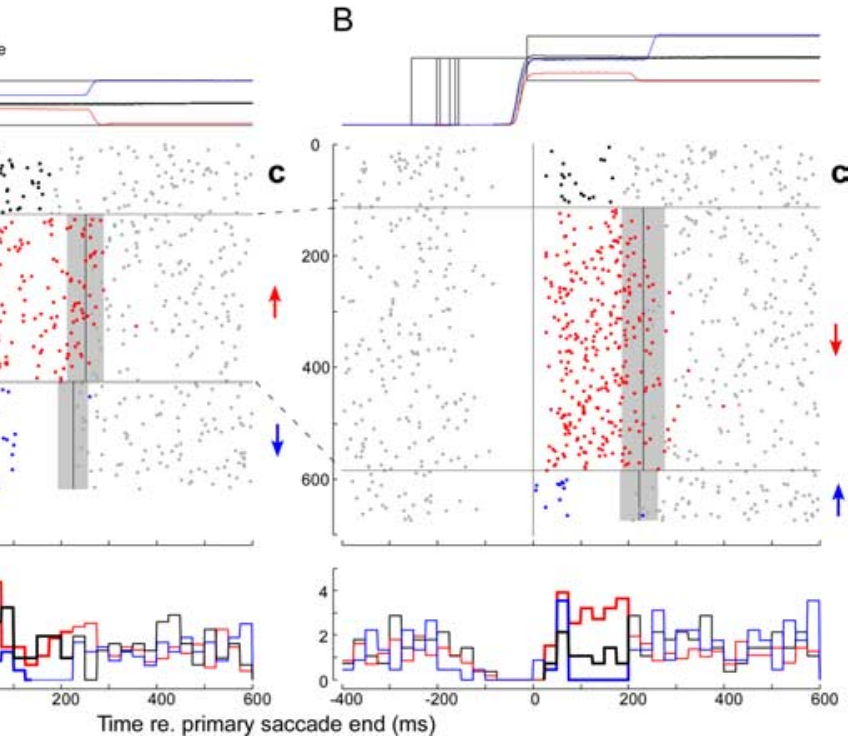

Time re. primary saccade end (ms)

Occurrence of CSs throughout each trial during different blocks of saccade adaptation for a representative P-cell. $A$, (he middle panels show the CSs (dots) associated with $>600$ trials in each direction plotted from top to bottom according blue). The gray bars indicate the SD s of the mean onset (solid vertical lines) of the corrective saccades within each adaptation block. correspond to those of CSs in error intervals above). For a complete explanation, see Results. This neuron was recorded at the center of the chamber, which was aligned along the midsagittal plane.

leftward and rightward saccade trials. Therefore, we feel confident about assigning the on- and off-direction errors for these 10 neurons. In three others $(2,7,8), \Delta \mathrm{CS}_{\text {block }}$ was positive for rightward errors and negative for leftward errors associated with leftward primary saccades. However, both leftward and rightward errors associated with rightward primary saccades caused a negative $\Delta \mathrm{CS}_{\text {block}}$. For the remaining neuron (11), the $\Delta \mathrm{CS}_{\text {block }}$ had the same sign for both error directions, although leftward errors produced a significantly lower $\Delta \mathrm{CS}_{\text {block }}$ than did the rightward errors ( $\mathrm{p} \ll 0.0001)$. We could not assign preferred error directions for these four neurons.

Of the remaining $13 \mathrm{P}$-cells, we could test 11 during backward adaptation in one direction and forward in the other (15-25). Based on the findings of the 10 unambiguous neurons above, we suggest that, when the same error associated with forward $(\uparrow)$ and backward $(\downarrow)$ adaptation leads to the same sign $\Delta \mathrm{CS}_{\text {block}}$, e.g., negative for unit 17 , the sign of the $\Delta \mathrm{CS}_{\text {block }}$ indicates either the on-direction (if positive) or off-direction (if negative, as for unit 17) error for the units. Therefore, we infer that we were testing the off-directions for units 16, 17, 19, 20, 22, and 23 and the on-directions of units 24 and 25 . Units 15 and 18 showed responses with opposite signs when the same direction error was created during leftward and rightward primary saccades. For both neurons, backward adaptation was associated with a positive $\Delta \mathrm{CS}_{\text {block }}$ and forward adaptation with a negative $\Delta \mathrm{CS}_{\text {block}}$. Therefore, we could not assign preferred error directions for these two units.

Finally, units 26 and 27, obtained early in our experimental series, were recorded during backward adaptation in both horizontal directions so that both rightward and leftward errors oc- 

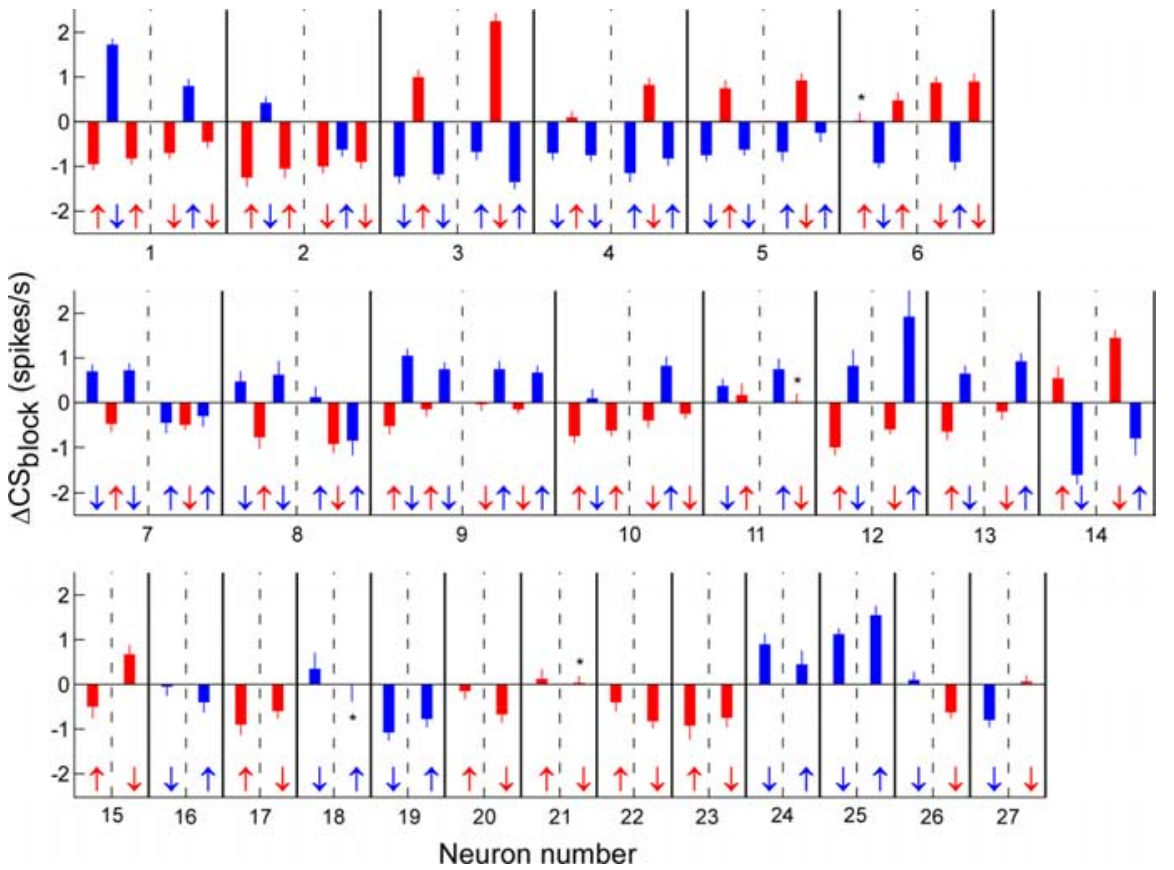

Figure 5. CS rate relative to baseline $\left(\Delta \mathrm{CS}_{\mathrm{block}} ; \mathrm{Eq} .3\right)$ during different adaptation blocks for all $27 \mathrm{P}$-cells. Each bar represents the $\Delta \mathrm{CS}_{\text {block }}$ from a different adaptation block. Bars to the left and right of the dashed lines are from leftward and rightward primary saccades, respectively. The error bar represents one SD computed from 1000 bootstrap samples (see Materials and Methods). Thick lines separate different P-cells. The upward and downward arrows represent forward and backward adaptation blocks, respectively. Blue identifies a rightward error, and red reflects a leftward error. These conventions will be used throughout the figures. Asterisks indicate data not significantly different from $0(p>0.05)$. The neuron illustrated in Figure 4 is neuron 14 .
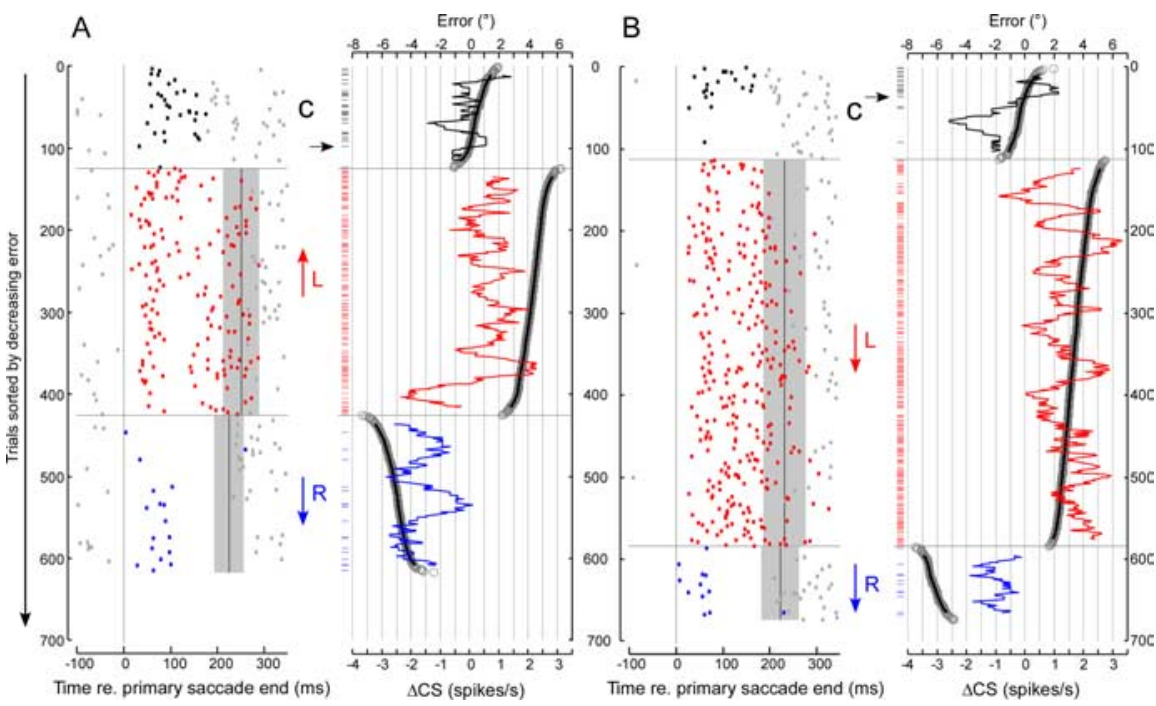

Figure 6. Comparison of running averages of the change of CS rate relative to baseline ( $\Delta C S)$ and of the error for the P-cell shown in Figure 4 with the trials sorted according to decreasing error amplitude. $\boldsymbol{A}$, Leftward primary saccades. $\boldsymbol{B}$, Rightward primary saccades. Left panels use the same data as those between -100 and $+350 \mathrm{~ms}$ in $A$ and $B$ of Figure 4 . Right panels show the 20 -trial running average of $C S$ rate relative to baseline within the intrasaccadic interval ( $\Delta C S ;$ Eq. 1) (fluctuating curves) and the error for each saccade (gray circles) as well as its 20-trial running average (Eq. 2) (thick black curve). Short horizontal tick lines (left of right panels in $\boldsymbol{A}$ and $\boldsymbol{B}$ ) show trials in which one or more $\mathrm{CS}$ occurs within the error interval. Horizontal arrows in $\boldsymbol{A}$ and $\boldsymbol{B}$ indicate when the error crosses 0 . Note that 0 error crossing coincides with the time when $C S$ suddenly stops firing. $C$, Control; $L$, leftward error; $\mathrm{R}$, rightward error.

curred. Unit 26 showed positive and negative $\Delta \mathrm{CS}_{\text {block }}$ for rightward and leftward errors, respectively, and vice versa for unit 27. Therefore, we conclude that units 26 and 27 preferred rightward and leftward error directions, respectively. In summary, we can unambiguously identify preferred directions in $10 \mathrm{P}$-cells and reasonably infer them in 11 others ( $78 \%$ of all neurons).
The preferred horizontal error direction of these 21 neurons generally reflected their recording sites. Relative to the center of the recording chamber, which was aimed at stereotaxic mediolateral 0 , $\mathrm{P}$-cells that preferred rightward error directions were recorded at mediolateral locations between left $1.15 \mathrm{~mm}$ and right $0.52 \mathrm{~mm}$ (median, left $0.46 \mathrm{~mm}$ ), whereas those that preferred leftward error directions were recorded between right 3.38 $\mathrm{mm}$ and the center (median, right 0.52 $\mathrm{mm}$ ). Therefore, the preferred error direction of these $21 \mathrm{P}$-cells was biased contraversive to their anatomical location.

\section{CS activity does not signal the magnitude of error Firing rate analysis} A qualitative examination of the data from the neuron illustrated in Figure 4 suggests that, when error is in the on-direction (shown in red), CS activity associated with backward $(\downarrow)$ and forward $(\uparrow)$ adaptation varies little during the course of adaptation although error is decreasing. Two different aspects of the data may mask a potential correlation between error and $\Delta$ CS. First, the error can vary substantially from one trial to the next, thereby possibly confounding a correlation between error and CS rate. To eliminate this variability, we sorted the data on the amplitude of the error as in Figure 6, a manipulation that produced a smooth change in error. Second, any trend in CS activity is difficult to discern because of the fluctuations in $\Delta \mathrm{CS}$ (Fig. 6). These fluctuations are not attributable to the conditions used to produce the running average because, when the number of trials contributing to the running average was increased from 20 to 50 and then to 100 (see Materials and Methods), the fluctuations remained at the same frequency but with reduced amplitudes. Moreover, the fluctuating behavior of $\Delta \mathrm{CS}$ is clearly visible in the modulation of the raw CS activity. For example, in Figure $6 B$, compare the fluctuations in the running average with the patterns of CSs, whose occurrences are shown by horizontal tic marks. We have no idea why the fluctuations occur, but they appear to be real and occur for every one of our P-cells.

Sorting the data according to error magnitude often revealed a sudden cessation of CS occurrences when the error crossed 0 (Fig. 6A, $B$, arrows). This suggests that $\mathrm{CS}$ occurrence is very sensitive to a change in error direction. In Figure 8, we explored this observation directly in four experiments in which we intentionally created conditions that caused the error to hover around 0 .

To quantify the relationship between $\Delta \mathrm{CS}$ and error, we per- 
formed a linear regression on data sorted according to decreasing error as in Figure 6. We considered only those data from adaptation blocks that caused a positive $\Delta \mathrm{CS}_{\text {block }}$ (Fig. 5). Therefore, there were 24 and 16 data blocks for backward and forward adaptation, respectively. The regression of the data in Figure 6, $A$ (trials 125-425) and $B$ (trials 113-584), yielded slopes of 0.43 and -0.30 with correlation coefficients of 0.31 and 0.30 , respectively. Therefore, these two linear regressions explained only $9 \%$ of the variance because of the substantial variability in the data.

For all of the data blocks collected during backward adaptation, approximately equal numbers of units had positive and negative slopes, which, for all but one, were $<0.4$ spikes $/ \mathrm{s} /{ }^{\circ}$. The population average was essentially $0\left(-0.011 \pm 0.27\right.$ spikes $\left./ \mathrm{s} /{ }^{\circ}\right)$. For forward adaptation, conversely, most regressions (14 of 16) had positive slopes. The population mean of the slope was $0.20 \pm$ $0.28 \mathrm{spikes} / \mathrm{s} /{ }^{\circ}$. Although the slopes of many of the correlations between $\Delta C S$ and error size were different from 0 , the correlation coefficients invariably were quite poor. Figure $7 A$ shows that the vast majority of correlation coefficients for data obtained during backward (92\%) and forward (69\%) adaptations were $<0.5$, indicating that the linear regressions accounted for $<25 \%$ of the variation in the data.

\section{Information theory analysis}

We grouped the same data according to error size and direction to build conditional probability (Eq. 4 ) tables. Table 1 shows data for the exemplar P-cell illustrated in Figure 4. The left entries were determined from data shown in red during backward adaptation in Figure $4 B$ for error bins centered at $+2,3$, and $4^{\circ}$. The right entries result from data derived from Figure $4 A$ (forward adaptations shown in red) for error bins surrounding +4 and $5^{\circ}$. We consider those trials in which a CS did occur $\left(\mathrm{CS}_{1}\right)$ and those in which it did not $\left(\mathrm{CS}_{0}\right)$ separately. If a CS occurred preferentially for a certain error condition, the entropy of the error would be less in the trials that a CS occurred. Conversely, if CS occurrence were not related to that error condition, then the entropy would be equal for the trials in which a CS did and did not occur.

For each error size, there was no consistent difference in the probability that a particular trial was or was not associated with a CS, for either backward or forward adaptation. The entropy of error when a CS occurred $\left[H\left(\right.\right.$ Error $\left.\left.\mid \mathrm{CS}_{1}\right)\right]$ and when it did not $\left[H\left(\right.\right.$ Error $\left.\left.\mid \mathrm{CS}_{0}\right)\right]$ (calculated from Table 1 , left) for the three errors obtained during backward adaptation was 1.52 and 1.47 bits, respectively. For three error sizes, the maximum possible entropy is 1.58 bits. The entropy for the two error sizes during forward adaptation (computed from Table 1, right) was 0.998 and 1.0 bits; the maximum possible entropy was 1.0 bit. Therefore, for this P-cell, there was no difference in the entropy of error size whether or not a CS occurred.

We constructed conditional probability tables for all of our $\mathrm{P}$-cells with sufficient data. We performed this analysis on adaptation blocks that showed both a directional preference and had a positive $\Delta \mathrm{CS}_{\text {block }}$ (Fig. 5). If two forward or two backward adaptation blocks each caused a positive $\Delta \mathrm{CS}_{\text {block}}$, e.g., see neuron 9, we combined all of the data from the same adaptation direction. Also, as mentioned in Materials and Methods, we did not consider blocks that provided $<50$ trials per bin. After all of these restrictions were met, we were able to analyze data from $17 \mathrm{P}$-cells in the backward and $12 \mathrm{P}$-cells in the forward adaptation condi-

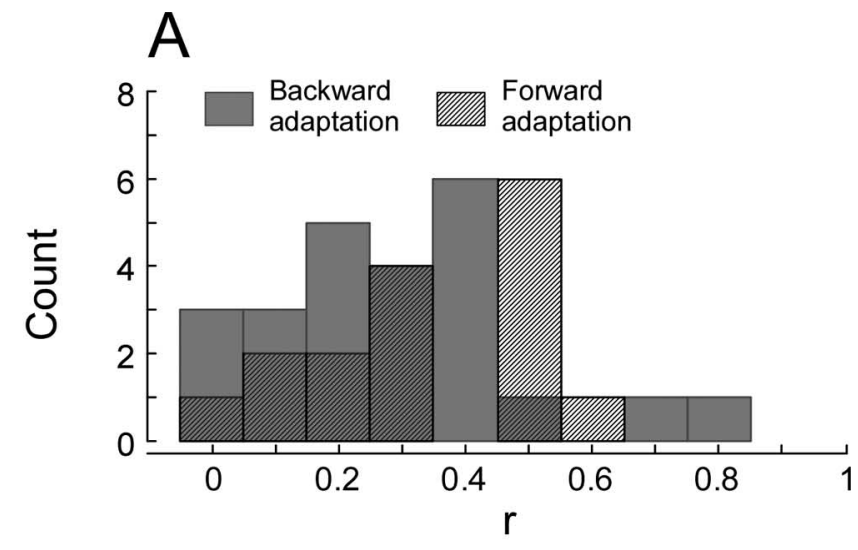

B

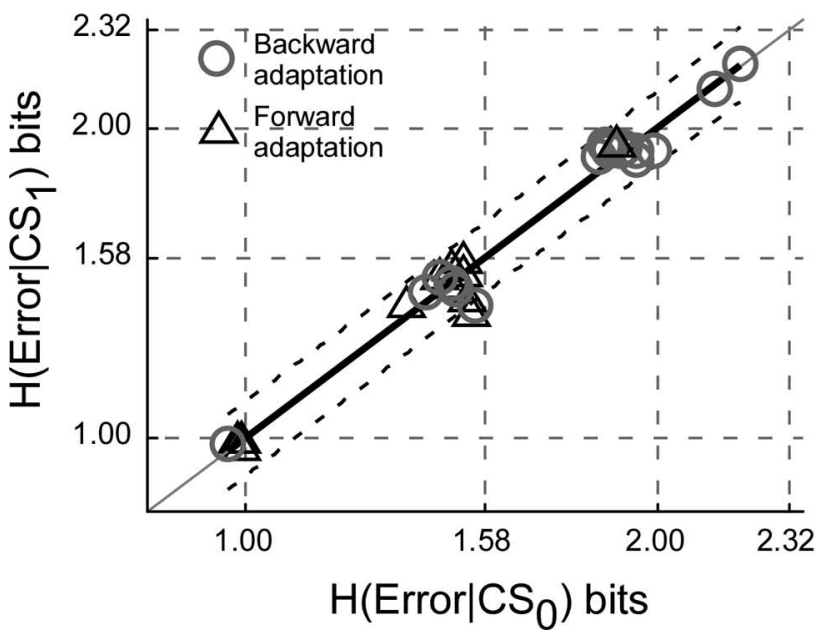

Figure 7. CS firing does not signal error size. $\boldsymbol{A}$, Distribution of regression coefficients of relationships between $\Delta C S$ and error size for backward and forward adaptation blocks. Almost all are $\leq 0.5$. B , Comparison of the entropy of error magnitude when a $\mathrm{CS}$ did $\left(\mathrm{CS}_{1}\right)$ and did not $\left(\mathrm{CS}_{0}\right)$ occur during forward and backward adaptation blocks. Dashed lines indicate $95 \%$ confidence intervals.

Table 1. Conditional probability tables of the exemplar unit shown in Figures 4 and 6

\begin{tabular}{|c|c|c|c|c|c|c|c|c|}
\hline \multicolumn{9}{|c|}{ Probability of trials for a certain error size } \\
\hline \multicolumn{5}{|c|}{ Backward adaptation } & \multicolumn{4}{|c|}{ Forward adaptation } \\
\hline Error size & $2^{\circ}$ & $3^{\circ}$ & $4^{\circ}$ & Total & Error size & $4^{\circ}$ & $5^{\circ}$ & Total \\
\hline $\mathrm{CS}_{0}$ & 0.16 & 0.40 & 0.44 & 1.00 & $\mathrm{CS}_{0}$ & 0.53 & 0.47 & 1.00 \\
\hline $\mathrm{CS}_{1}$ & 0.21 & 0.44 & 0.35 & 1.00 & $\mathrm{CS}_{1}$ & 0.50 & 0.50 & 1.00 \\
\hline
\end{tabular}

tions. Of these 29 analyses, four had two error sizes ( 1 bit maximum), 13 had three error sizes ( 1.58 bits maximum), 10 had four error sizes ( 2 bits maximum), and two had five error sizes $(2.32$ bits maximum).

Figure $7 B$ compares the entropy when a CS occurred during the error interval and when it did not. All data, whether obtained from forward (triangles) or backward (circles) adaptations, lie very near the line of slope 1.0. The regression of the data had a slope of 1.01 , an intercept of -0.01 , and a correlation coefficient of 0.99 . All but two points lay within the $95 \%$ confidence interval (dashed lines). Because the entropies of error are the same whether a CS occurred in the error interval or not, the occurrence of a CS does not report the size of the error.

Therefore, the results of both the firing rate and information 

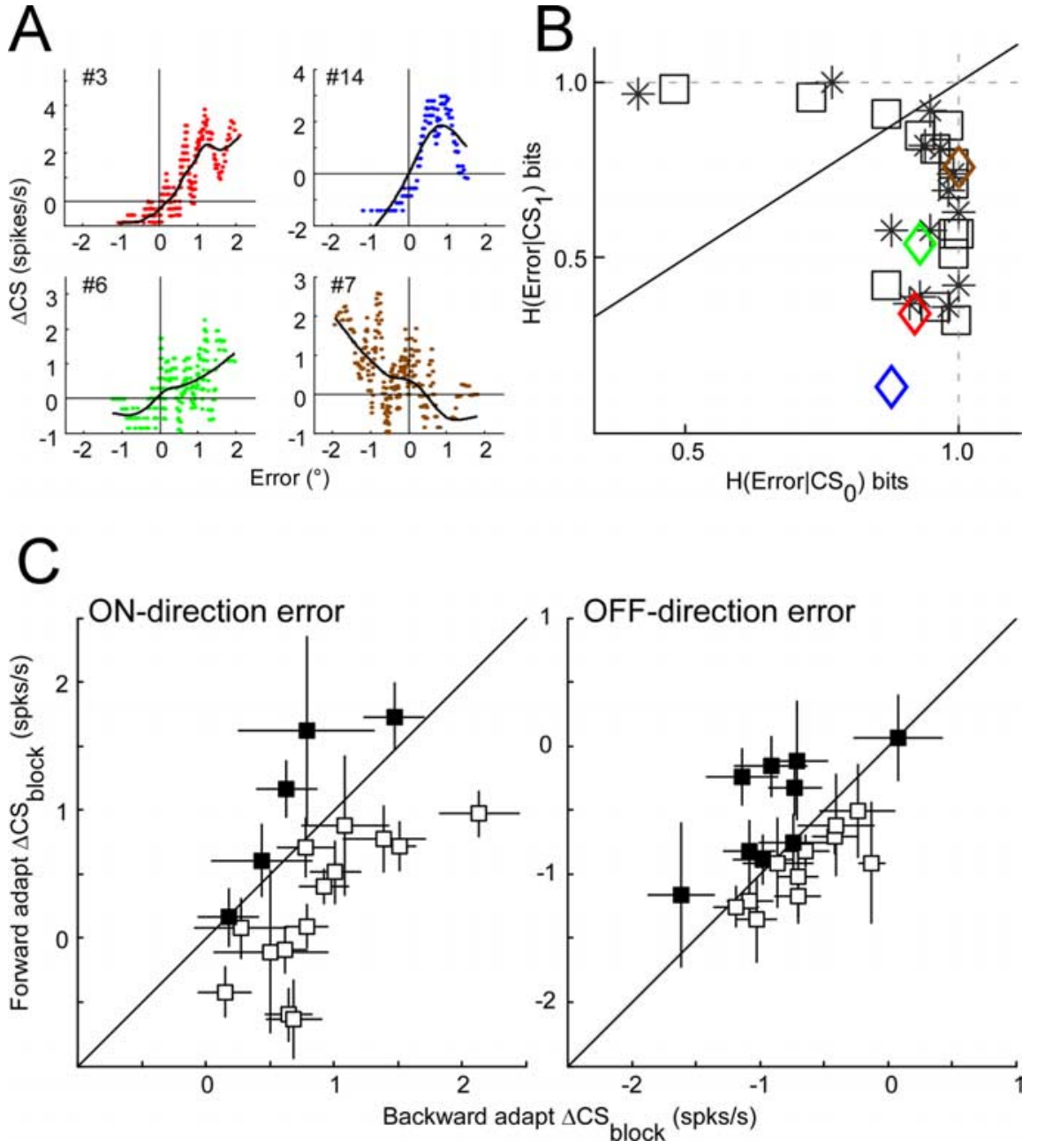

Figure 8. CS firing does signal error direction. $\boldsymbol{A}$, Relationship between $\Delta \mathrm{CS}$ and error size when the error was made to hover around 0 (see Results) for four neurons during backward adaptation. Horizontal and vertical lines indicate 0 spikes $/ \mathrm{s}$ and $0^{\circ}$ error. Curves are cubic spline fits. $\boldsymbol{B}$, Comparison of the conditional entropy of error direction when a $C S \operatorname{did}\left(\mathrm{CS}_{1}\right)$ and did not $\left(\mathrm{CS}_{0}\right)$ occur. Squares, asterisks, and diamonds represent data from backward adaptation, forward adaptation, and the four experiments shown in $A$, respectively. Black line has a slope of 1.0. C, Comparison of $\Delta \mathrm{CS}_{\text {block }}$ for forward and backward adaptations with comparable error amplitudes. Each point represents data from a single P-cell. The error bar represents one SD computed from 1000 bootstrap samples (see Materials and Methods). On- and off-direction errors are considered separately.

Table 2. Conditional probability tables of the exemplar unit shown in Figures 4 and 6

\begin{tabular}{|c|c|c|c|c|c|c|c|}
\hline \multicolumn{8}{|c|}{ Probability of trials for error direction } \\
\hline \multicolumn{4}{|c|}{ Backward adaptation } & \multicolumn{4}{|c|}{ Forward adaptation } \\
\hline Error direction & Right & Left & Total & Error direction & Right & Left & Total \\
\hline $\mathrm{CS}_{0}$ & 0.47 & 0.53 & 1.00 & $\mathrm{CS}_{0}$ & 0.33 & 0.67 & 1.00 \\
\hline $\mathrm{CS}_{1}$ & 0.06 & 0.94 & 1.00 & $\mathrm{CS}_{1}$ & 0.07 & 0.93 & 1.00 \\
\hline
\end{tabular}

theory analyses support our conclusion that CS activity does not signal the magnitude of eye-position error.

\section{CS activity does signal the direction of error}

Firing rate analysis

Figure 5 shows that the sign of $\Delta \mathrm{CS}_{\text {block }}$ is a reliable indicator of the direction or sign of the error in almost all of our P-cells. However, in those datasets, most adaptations did not drive the eye-position error all the way to 0 , so the error remained either positive or negative. For four P-cells, we held isolation long enough to be able to adjust the amount of the intrasaccadic target back step so that the resulting eye-position error was 0 , on average, but varied from slightly positive to slightly negative on different trials. With these data sorted on error amplitude, the plots of the running averages of $\Delta C S$ versus error (Fig. $8 A$ ) showed that, when the error was at 0 , the $\Delta$ CS also was near 0 for all four P-cells Moreover, the $\Delta \mathrm{CS}$ changed suddenly from positive to negative when the error changed sign. These data illustrate convincingly that the sign of CS activity is very sensitive to the direction, i.e., leftward or rightward, of eye-position error.

\section{Information theory analysis}

We also measured the entropy of the error to determine how well CSs signal error direction. To do so, we considered data for which the direction of adaptation was the same but the error direction was different. For example, with our exemplar P-cell (Fig. 4), we determined the conditional probability (Eq. 4) by combining the red data in $A$ with the blue data in $B$ for forward adaptation (Table 2, right column) and the blue data in $A$ with the red data in $\mathrm{B}$ for backward adaptation (Table 2, left column). When a CS occurred, there was a substantial probability that the error was leftward for both forward and backward adaptation. When a CS did not occur, the preferred direction was less clear. The entropies of error direction when a CS did occur $\left[H\left(\right.\right.$ Error $\left.\left.\mid \mathrm{CS}_{1}\right)\right]$ and did not $\left[H\left(\right.\right.$ Error $\left.\left.\mid \mathrm{CS}_{0}\right)\right]$ were 0.32 and 1.00 , respectively, for backward adaptation and 0.38 and 0.91 for forward adaptation. The lower entropies of error direction when a CS did occur indicate that the occurrence of a CS provided information about error direction.

Because this analysis required horizontal errors in both directions for either forward or backward adaptation, we could perform it only on the $14 \mathrm{P}$-cells for which we reversed the direction of adaptation. For all $14 \mathrm{P}$-cells, $H$ (Error $\mid \mathrm{CS}_{1}$ ) was lower than $H$ (Error $\mid \mathrm{CS}_{0}$ ) for 11 neurons during backward and 12 during forward adaptation (Fig. $8 B$ ). The average $H\left(\right.$ Error $\left.\mid \mathrm{CS}_{1}\right)$ and $H\left(\right.$ Error $\left.\mid \mathrm{CS}_{0}\right)$ during backward adaptation was $0.69 \pm 0.23$ and $0.91 \pm 0.14$, respectively, and during forward adaptation was $0.66 \pm 0.22$ and $0.90 \pm 0.15$, respectively. For both forward and backward adaptation, $H$ (Error $\mid \mathrm{CS}_{1}$ ) was significantly less than $H\left(\right.$ Error $\left.\mid \mathrm{CS}_{0}\right)$ (paired $t$ test, $p<0.05)$. This was true even when the error was small, as was the case for the subset of data shown in Figure $8 \mathrm{~A}$. For each of those neurons, $H$ (Error $\left.\mid \mathrm{CS}_{1}\right)$ was significantly smaller than $H$ (Error $\mid \mathrm{CS}_{0}$ ) [color-coded diamonds in Figure $8 \mathrm{~B}$ ]. The average $H\left(\right.$ Error $\left.\mid \mathrm{CS}_{1}\right)$ and $H\left(\right.$ Error $\left.\mid \mathrm{CS}_{0}\right)$ for all four P-cells were $0.44 \pm$ 0.27 and $0.93 \pm 0.05$, respectively. Finally, $H\left(\right.$ Error $\left.\mid \mathrm{CS}_{1}\right)$ for all 14 neurons was not significantly different for forward and backward adaptation (paired $t$ test, $p>0.5$ ). 


\section{Does CS response to error direction} vary with the adaptation condition? Although information theory showed that the CS encodes error direction equally well for forward and backward adaptation, the magnitude of the CS rate might not be equal. For 14 neurons, we could compare the change in CS activity for equivalent error sizes in both the on- and off-directions associated with backward and forward adaptations. Therefore, these neurons provided 28 comparisons, 14 each for the on- and off-direction. For the remaining 11 other units, we could compare CS responses for equivalent errors in either the on-direction $(n=5)$ or offdirection $(n=6)$ that led to forward and backward adaptation. Therefore, we could perform a total of 19 and 20 comparisons for on- and off-direction errors, respectively. The number of saccades per block used to compute $\Delta \mathrm{CS}_{\text {block }}$ in this analysis ranged from 28 to 493 (median, 127).

Figure $8 C$ compares the $\Delta \mathrm{CS}_{\text {block }}$ for errors in the on-direction and offdirection associated with forward and backward adaptations. For on-direction errors, $\Delta \mathrm{CS}_{\text {block }}$ of 14 of $19 \mathrm{P}$-cells (open squares) during backward adaptation was significantly higher than that during forward adaptation (one-tailed unpaired $t$ test, $p<0.0001$ ). For the remaining five, the $\Delta \mathrm{CS}_{\text {block }}$ was not significantly higher $(p>0.07)$. The median differences of $\Delta C S-$ block for all 19 was 0.52 spikes/s (range, $-0.84-1.32$ ), which is significantly different from 0 (sign test, $p<0.02$ ). For offdirection errors, $\Delta \mathrm{CS}_{\text {block }}$ of 11 of $20 \mathrm{P}$-cells was significantly greater for backward than for forward adaptation (one-tailed unpaired $t$ test, $p<0.0001)$. However, the range of the differences of $\Delta \mathrm{CS}_{\text {block }}$ (backward - forward) of all 20 neurons was $-0.90-$ 0.79 spikes/s, and the median difference $(0.061$ spikes/s $)$ was not significantly different from 0 (sign test, $p>0.2$ ). Therefore, for errors in the on-direction, backward adaptation is associated with a greater increase in CS activity than is forward adaptation in most P-cells. For errors in the off-direction, the decrease in CS activity associated with backward and forward adaptation is similar across our population of P-cells.

\section{Is the change in CS activity during the error interval actually related to the corrective saccade?}

Because the increase (or decrease) in CS rate occurs in the intersaccadic interval, it conceivably could be related to the ensuing corrective saccade and not just the error. To test this possibility, we compared the CS discharge associated with $\sim 5^{\circ}$ control saccades, which were chosen to be similar to the corrective saccades early in adaptation, with the CS discharge associated with forward and backward corrective saccades in the same direction during adaptation. We limited the size of the saccades to $3.5-6.5^{\circ}$, a range that includes both control and corrective saccades. Figure 9 shows CS activity associated with $\sim 5^{\circ}$ rightward saccades in three different conditions: to rightward control target steps $\left(A_{1}\right.$ and $\left.B_{1}\right)$ and to rightward adapt steps during forward $\left(A_{2}\right.$ and $\left.B_{2}\right)$ and backward ( $A_{3}$ and $B_{3}$ ) adaptation (Fig. 5, unit 1).

We compared the activity occurring between the primary and corrective saccades during adaptation with the activity between the target step and the $5^{\circ}$ saccade during the control trials. In the $B$ column, the CS dot rasters for each trial are aligned on the onset of $5^{\circ}$ saccades (see schematic examples on the right) and sorted from top to bottom according to either the reaction time of control saccades $\left(B_{1}\right)$ or the intersaccadic interval between the primary and corrective saccade $\left(B_{2}\right.$ and $\left.B_{3}\right)$ in adaptation trials. The curve of small gray dots indicates the time of occurrence of either the target step or the end of the primary saccade. The incidence of CSs within the error interval of both adaptation conditions and a comparable period for control saccades increased in all three cases, with the most prominent increase associated with backward adaptation (histograms, $B_{4}$ ).

In the $A$ column, data are aligned on the target step to illustrate where we determined the baseline activity over the $175 \mathrm{~ms}$ preceding the target step to calculate $\Delta \mathrm{CS}_{\text {block }}$ in Equation 3. For this P-cell, $\Delta \mathrm{CS}_{\text {block }}$ was $1.48 \pm 0.12,0.85 \pm 0.14$, and $0.45 \pm 0.36$ spikes/s for backward and forward adaptation and the control, respectively. During backward adaptation, $\Delta \mathrm{CS}_{\text {block }}$ was $74 \%$ higher than for forward adaptation and 228\% higher than for control saccades. During forward adaptation, $\Delta \mathrm{CS}_{\text {block }}$ was $89 \%$ higher than for control saccades.

Figure 10 compares the $\Delta \mathrm{CS}_{\text {block }}$ across all 10 neurons tested (neuron from Fig. 9 is neuron 1). When the adaptations were produced by errors in the on-direction (Fig. $10 A$ ), the $\Delta \mathrm{CS}_{\text {block }}$ during backward adaptation (black bar) was always significantly $(p \ll 0.0001)$ greater than that for either control saccades (open bar) or forward adaptation (gray bar). For some P-cells, $\Delta \mathrm{CS}_{\text {block }}$ was negative during control and/or forward adaptation conditions. The $\Delta \mathrm{CS}_{\text {block }}$ during forward adaptation was significantly higher $(p<0.05)$ than that associated with $5^{\circ}$ control saccades for only three neurons $(1,6,14)$. 

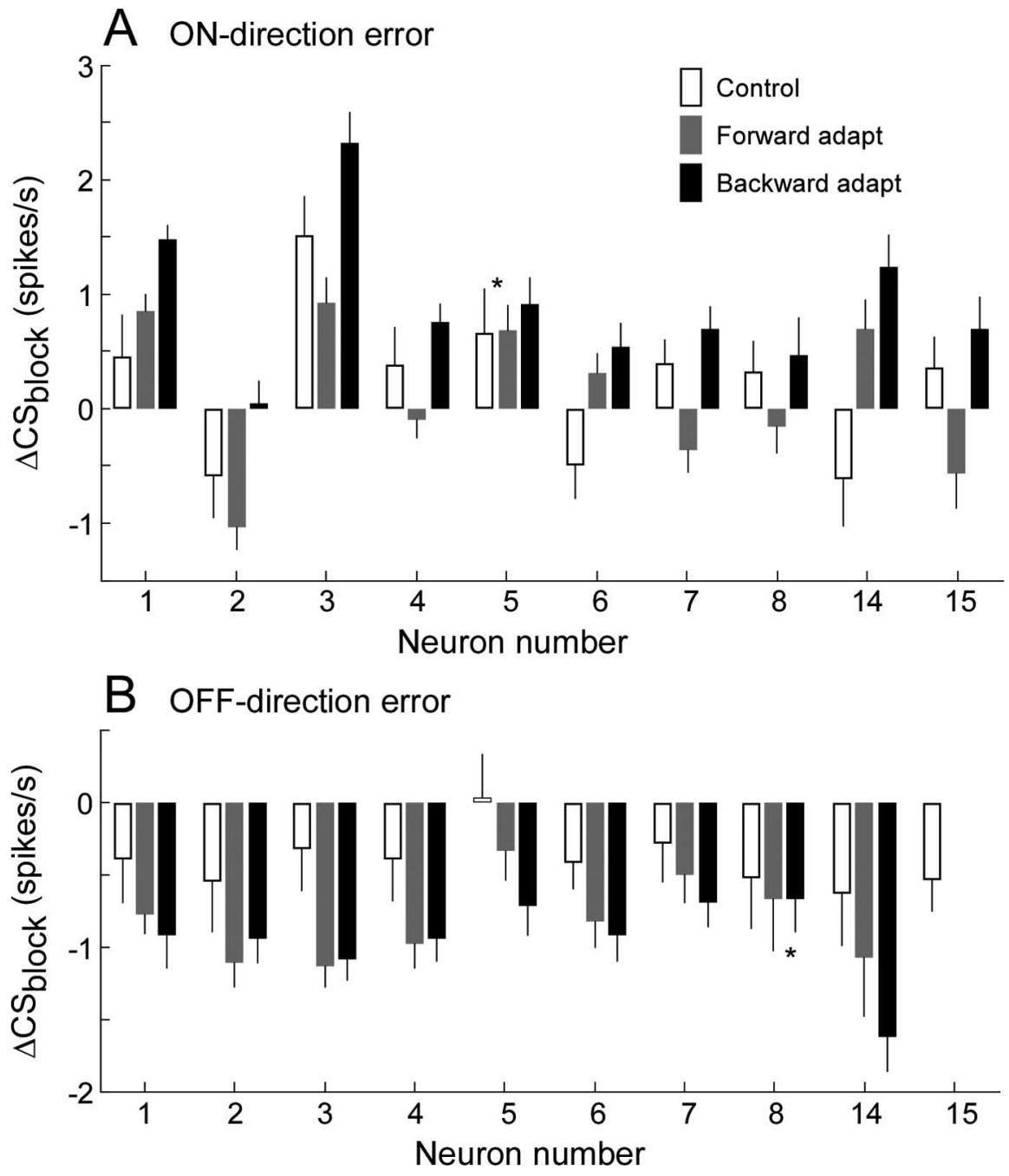

Figure 10. Comparison of $\Delta \mathrm{CS}_{\text {block }}$ during control and forward and backward adaptation blocks calculated from data like those in Figure 9 for that neuron and nine others. $\boldsymbol{A}, 0 \mathrm{n}$-direction error; $\boldsymbol{B}, 0$ ff-direction error. The error bar represents one SD computed from 1000 bootstrap samples (see Materials and Methods). Asterisk indicates that means are not significantly different $(p>0.05$, unpaired $t$ test). Neuron numbering is the same as in Figure 5 .

When adaptation was produced by errors in the off-direction of a unit (Fig. $10 \mathrm{~B}$ ), CSs during control $5^{\circ}$ saccades occurred less frequently than in baseline conditions, i.e., $\Delta \mathrm{CS}_{\text {block }}$ was negative, for all 10 neurons except neuron 5 . However, when $5^{\circ}$ saccades were associated with either backward or forward adaptations, $\Delta \mathrm{CS}_{\text {block }}$ was even more negative for all nine neurons tested $(p<$ $0.0001)$. Neuron 15 died before we could test adaptation in the off-direction. The number of saccades per block used to compute $\Delta \mathrm{CS}_{\text {block }}$ in the analysis of Figure 10 ranged from 60 to 862 (median, 178).

In summary, our data indicate that the change in CS firing during the error interval of adaptation trials is not solely the result of a change in activity associated with the corrective saccade itself. When errors in the on-direction drove backward adaptation, the associated change in CS activity always was statistically greater than that associated with similar control saccades. Moreover, this analysis confirms our previous conclusion that increases in CS firing associated with backward adaptations are greater than those associated with forward adaptations. For off-direction errors, the decreases in firing accompanying forward and backward adaptation are more similar but also are greater than those accompanying control saccades.
Pattern of increased CS activity

For the on-direction error, the CSs of some of our P-cells appeared to occur in bursts within the error interval. To document how frequently this occurred, we aligned trials with on-direction errors on the end of the primary saccade and calculated the running average of CS probability within a $30 \mathrm{~ms}$ window that slid throughout the error interval. The running average started from $50 \mathrm{~ms}$ before the end of the primary saccade to $50 \mathrm{~ms}$ after the mode of the end of the error interval. To minimize the effect of variable error intervals, we calculated the mode of the error interval separately for each block of data with $5 \mathrm{~ms}$ resolution and included only trials with intervals within $\pm 5 \mathrm{~ms}$ of the mode. For different adaptation blocks, the mode of the error interval ranged from 144 to 204 ms. Finally, we considered only those adaptation blocks in which at least 40 (range from 40 to 353; median, 79) trials remained after limitation of the error interval. After these restrictions, $14 \mathrm{P}$-cells could be analyzed during backward adaptation and 12 during forward adaptation.

Figure 11 shows running averages of CS probability for two exemplar P-cells during backward adaptation. Like the neuron in Figure $11 A$ (Fig. 5, neuron 4), many P-cells showed a bimodal or multimodal distribution of CSs in the error interval. However, only 2 of 353 trials had multiple spikes in the error interval. Therefore, the multimodal distribution was the result of a single CS occurring at different latencies (range of $0-200 \mathrm{~ms}$ ) on different trials. Like the neuron in Figure $11 B$ (Fig. 5, neuron 5), the distribution of CSs in the error interval was unimodal for other neurons.

The distributions of CSs for the on-direction error during backward adaptation are shown in Figure $11 C$ for all 14 neurons with sufficient data. We arranged their order loosely according to the qualitative robustness of multimodal activity. The majority has multimodal responses. A similar display for the 12 neurons with on-direction errors produced during forward adaptation (Fig. 11D) also reveals some clear multimodal distributions; however, a multimodal distribution was more difficult to detect during forward adaptation because there were fewer trials. The distance between the first two peaks of multimodal distributions during both backward (first 12 P-cells) and forward (first 9 P-cells) adaptations ranged from 39 to 116 and 47 to $140 \mathrm{~ms}$, with averages of $72.08 \pm 29.34$ and $86.44 \pm 31.29 \mathrm{~ms}$, respectively. These averages are not significantly different $(p>0.1)$.

Finally, we estimated the latency of the occurrence of a CS relative to the end of primary saccade as the time when the running average of CS probability first reached half of its peak between $t=0$ and $250 \mathrm{~ms}$ (Fig. $11 A, B$, vertical lines in the bottom panels). For the neurons in Figure 11, $A$ and $B$, the latencies were 43 and $96 \mathrm{~ms}$, respectively. On average, across all P-cells, the latencies of CS occurrences during backward and forward adap- 
tation in the on-direction error were $68 \pm$ 42.2 and $65.6 \pm 28.8 \mathrm{~ms}$, respectively.

\section{CS pauses during primary saccades}

As we saw in Figure 4, CS activity also exhibits a pause with saccades, whether the saccades are part of an adaptation trial or not. To document the relationship of pauses to saccades, we considered just the primary saccades in the adaptation trials for two reasons. First, there were many more trials in the adaptation blocks so we could obtain a reasonable spike density histogram with a narrower Gaussian function (see Materials and Methods). Second, some control saccades overshoot and some undershoot the target, creating either a positive or negative error. We have seen that a change in the sign of the error leads to a change in the sign of $\Delta \mathrm{CS}$; this could confound the determination of pause timing. For example, in Figure $6 B$ (trials 1-112), the control trials with negative errors (trials $\sim 50-100$ ) were associated with a cessation in CS firing, which would give rise to the erroneous impression that the pause associated with these hypometric saccades ended at $+200 \mathrm{~ms}$ rather than at less than $+50 \mathrm{~ms}$, which is the case for saccades that are followed by positive errors. Therefore, we examined only those 19 P-cells for which the position error at the end of the primary saccades was in the on-direction, i.e., was associated with an increase in activity.

The pause in activity was different if the primary saccades were contraversive (backward adapt trials) or ipsiversive (forward adapt trials) to the on-direction error. During primary saccades contraversive to the on-direction error, the CS activity of all 19 units paused. The pause of all but one began before the saccade and, in 18 of 19, began between 16 and $116 \mathrm{~ms}$ before the saccade (median entire population, $-81 \mathrm{~ms}$; mean, $-68.89 \pm 36.57 \mathrm{~ms}$ ). The pause lasted an average of $33.36 \pm 22.51 \mathrm{~ms}$ beyond the end of the saccade. Therefore, the average pause duration $(154 \pm 48.23 \mathrm{~ms})$ was much greater than the duration of an average $12-15^{\circ}$ saccade, whose shortest and longest durations were $42.58 \pm 3.77$ and $68.77 \pm 16.03 \mathrm{~ms}$, respectively.

During primary saccades ipsiversive to the on-direction error, 17 of 19 neurons also exhibited pauses in CS activity. The pause began either well before $(-110.83 \pm 17.84 \mathrm{~ms} ; n=6)$ or mostly after $(+19.64 \pm 21.35 \mathrm{~ms} ; n=11)$ saccade onset. For those six neurons whose pause began well before ipsiversive saccade onset, pause duration was $\sim 28 \mathrm{~ms}$ longer than the pause duration associated with their associated contraversive saccades $(p<0.05)$. For those 11 whose pauses began mostly after saccade onset, the pause lasted, on average, $35.18 \pm 36.46 \mathrm{~ms}$ beyond the end of the saccade; the average pause duration was $73 \pm 42.58 \mathrm{~ms}$. Finally, there was no consistent relationship between pauses in CS activity and SS activity. For example, four of the six P-cells with long pauses in CS activity for both ipsiversive and contraversive saccades discharged a burst of SSs for those saccades, whereas the remaining two showed pauses.

A pause also occurred during $5^{\circ}$ saccades in the 10 neurons for which they were tested. The pause duration related to $15^{\circ} \mathrm{sac}-$ cades was significantly longer than for $5^{\circ}$ saccades $(p<0.05)$. For $5^{\circ}$ saccades, the pause started $\sim 36-63 \mathrm{~ms}$ later, on average, than for $15^{\circ}$ saccades $(p<0.05)$, although the pause ended at approximately the same time $(p>0.5)$. Pause duration was also longer for contraversive than ipsiversive $5^{\circ}$ saccades $(p<0.05)$.

In summary, all P-cells showed phasic changes of CS firing for saccades. For 5 or $15^{\circ}$ saccades contraversive to the preferred error direction of the neuron, the accompanying CS pause was longer and started earlier than that for ipsiversive saccades. For both ipsiversive and contraversive saccades, larger saccades were associated with longer pauses. However, for most P-cells, pause duration exceeded saccade duration for contraversive (24 of 25 neurons) and ipsiversive (18 of 25) saccades. 


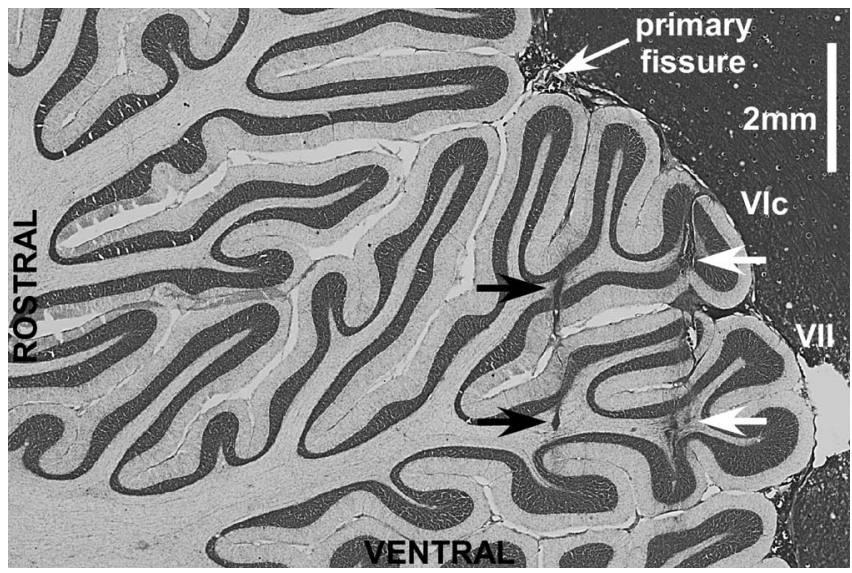

Figure 12. Parasagittal section of the vermis with the oculomotor folia, lobules VIc and VII, clearly shown. Black arrows indicate the location of electrolytic lesions in which hot saccaderelated activity was recorded. White arrows point to more dorsal electrode tracks. Dark material surrounding the cerebellum is egg yolk.

\section{Site of the recordings}

The location of recorded P-cells was confirmed in one monkey. In one penetration, we made two electrolytic lesions that straddled the region in which we found a high density of saccaderelated burst activity. Figure 12 shows a parasagittal section $(\sim 0.5$ $\mathrm{mm}$ right of the stereotaxic 0 ) of the oculomotor vermis showing the electrolytic lesions (black arrows). The lesions are located caudal of the primary fissure that separates lobules V and VI. Previous studies have demonstrated that the oculomotor vermis is located in lobules VIc and VII [Noda and Fujikado (1987), their Fig. 8]. Gliosis caused by other, more posterior electrode tracks (white arrows) also was located in both the posterior part of lobule VI and lobule VII.

\section{Discussion \\ Complex spike activity changes during saccade amplitude learning}

Both our firing rate and information theory analyses demonstrated that complex spike activity in the oculomotor vermis changes when a monkey experiences a behaviorally induced saccade dysmetria. The change of CS activity was confined only to the interval between the primary and corrective saccade when there is an error in eye position present.

The firing rate analysis implies that CS activity signals the direction but not the amount of the error. When the error exhibited a sign reversal either naturally during control conditions (Fig. 6A, $B$, arrows) or when we deliberately tried to null the error (Fig. $8 A$ ), there was an immediate change in the sign of CS activity. These data suggest that the sign of changes in CS firing relative to baseline activity was very sensitive to the direction of the error. In contrast, the change in CS rate was poorly correlated, if at all, with the magnitude of the error. The information theory analysis confirmed these two conclusions. The entropy of error size was not different whether a CS occurred or not. However, the entropy of error direction was significantly lower when a CS occurred. CS activity also exhibits a selectivity for target error direction during reaching tasks (Kitazawa et al., 1998) and for stimulus movement during the ocular following response (Kobayashi et al., 1998) and smooth pursuit (Stone and Lisberger, 1990).

In addition to the change in CS activity during the error interval, all P-cells exhibited a pause in firing whether the saccade was associated with an adaptation trial or not. This unexpected finding indicates that CS firing may have a separate role in the generation of "ordinary" saccades.

\section{Is the $\mathrm{CS}$ response during the error interval visual?}

The average latency of CS activity from the end of primary saccades was $\sim 70 \mathrm{~ms}$, which is similar to visual response latencies in other subcortical visual structures, e.g., the superior colliculus and the pretectum (e.g., Mays and Sparks, 1980). However, the CS response associated with saccade adaptation is not a "naive" visual signal because it is larger when associated with a decrease than an increase in saccade amplitude. The CSs of 14 of our 19 tested P-cells discharged at significantly $(p<0.0001)$ higher rates for backward than forward adaptation (by a median of 0.62 spikes/s). These differences in CS modulation may be related to the observation that backward adaptation progresses more rapidly and is more robust than forward adaptation (Straube et al., 1997).

CS activity associated with other forms of motor learning also is different for adaptation that causes amplitude or velocity increases or decreases. During adaptation of the vestibulo-ocular reflex, CSs in P-cells of the flocculus respond differentially for gain increases and decreases, at least at high frequencies of oscillation (Watanabe, 1984; Raymond and Lisberger, 1998). Also for smooth pursuit learning, CS activity in the flocculus changes for adapted decreases in acceleration but not increases (Kahlon and Lisberger, 2000). Therefore, during adaptation of both reflexive and precision oculomotor behaviors, CS discharge reflects the sign of the adaptation.

An interesting feature of activity within the error interval is that the CSs of many P-cells tend to have a multimodal distribution, on average, relative to the end of the primary saccade (Fig. 11). This observation suggests that the inferior olive may have an inherent rhythmicity that is synchronized by the occurrence of the saccade. A similar rhythmic discharge of CSs, but at a lower frequency, occurs with forepaw stimulation in cats (Bloedel and Ebner, 1984).

\section{Do changes in CS activity drive saccade adaptation?}

If the complex spikes guide saccade learning, they should occur more frequently under conditions that drive adaptation. Our data cannot resolve this issue directly. However, the results obtained from 10 neurons, which we tested using the same error in two different behavioral conditions, suggest that changes in CS activity are associated with saccade adaptation. In one condition, the error required the animal to make backward corrective saccades (Figs. 9, 10) and in the second to make the same size saccade to a simple target step. In the first error condition, which produced adaptive reduction of saccade amplitude, CSs discharged at significantly higher rates than in the second condition for all 10 neurons. Therefore, these data imply that the change in CS activity does drive saccade adaptation.

Why is the size of the error not reported along with its direction? Perhaps the adaptation process requires only a binary error signal that indicates whether to drive an increase or decrease in saccade amplitude. In this scenario, CSs would keep reporting that there was an error signal until adaptation was complete. Indeed, in the four experiments in which we reduced back-step sizes to simulate the completion of adaptation, the change in CS activity disappeared as soon as the error reached 0 . Such a binary (bang-bang) control mechanism is attractive because the small error introduced by the natural variability in the accuracy of normal saccades (see control data in Fig. 6) would constantly 
signal whether saccades were staying accurate. Consistent with this suggestion, Robinson et al. (2003) recently showed that smaller errors are more efficient at driving adaptation than are large ones. Finally, a model that uses an error signal that indicates only the direction of saccade dysmetria is sufficient to simulate the general properties of saccade adaptation (Dean et al., 1994). Of course, it also is possible that a signal about error magnitude is available from another source.

We acknowledge that a simple model for adaptation that involves only plasticity at the P-cell is probably very naive. In the vestibulo-ocular reflex, plasticity apparently occurs not only at the flocculus P-cell but also at its target cells in the vestibular nucleus (Miles and Lisberger, 1981). Moreover, in the smooth pursuit system, although appropriate changes in CS activity occur during backward adaptation (Kahlon and Lisberger, 2000), there are no concomitant changes in SS activity as proposed in the models by Marr (1971) and Albus (1971). Therefore, Kahlon and Lisberger (2000) conclude that smooth pursuit plasticity occurs before the cerebellum. Unfortunately, our adaptation paradigm produced changes in saccade amplitude that were too small to allow a quantitative assessment of the expected changes in P-cell SS timing with saccade duration (Ohtsuka and Noda, 1995).

Complex spike activity also changes in the appropriate cerebellar P-cells during adaptation of somatomotor movements. When monkey wrist movements were subjected to unexpected load changes, CS activity initially increased and gradually returned to baseline as the movement adapted to the new load (Gilbert and Thach, 1977). Because P-cells also showed gradual changes in SS activity, this study could support the models of Marr and Albus. In an "indirect" reaching task, changing the gain of a manipulandum and the visual cursor it controlled caused an increase in CS activity, which did not change with adaptation (Ojakangas and Ebner, 1992). These data, which resemble ours, suggest that CS activity might report the direction but not the magnitude of the error.

\section{CS activity during saccade motor learning in other studies}

In another study that appeared when ours was being reviewed, Catz et al. (2005) reached substantially different conclusions. Using similar behavioral adaptations of saccade size, they reported that, for backward adaptation, CS discharge increased as saccade amplitude decreased in their population average. In contrast, for forward adaptation, a pause in CS firing materialized early in adaptation and gradually grew in duration as saccade amplitude increased. Because these changes continued after adaptation was complete, i.e., "when the error had been nulled," they suggested that CS firing underlay the stabilization of the adapted saccade rather than serving as an error signal. In contrast, when the error was 0 in our data, the change in CS also was 0 . Moreover, their changes in CS rate, either an increase for backward or a decrease for forward adaptation, reached a maximum for their neuronal population before the primary saccade was over. In contrast, the first CS for our cells occurred in the intersaccadic interval $\sim 70 \mathrm{~ms}$ after the end of the primary saccade. Their finding that the maximum CS activity at the end of the saccade is especially perplexing because all of our primary saccades were accompanied by a pause in firing (histograms in Fig. 4). Moreover, Catz et al. (2005) state, "there was no noticeable saccade-related CS modulation of the population before the onset of either inward or outward adaptation."

It is difficult to reconcile two studies with such obvious qualitatively different results. One major methodological difference is that we demonstrate our findings on each individual P-cell rather than on the entire population in which one especially responsive neuron might dominate the average [Catz et al., (2005), their Fig. $3 C]$. Also, because CS rate is very sensitive to the sign but not the magnitude of the error, their finding that CS activity was maintained when error apparently had been eliminated might be explained if an error, however small, still remained. Clearly, it will be important to resolve the discrepancies between these two studies.

\section{References}

Albus J (1971) A theory of cerebellar function. Math Biosci 10:25-61.

Bahill AT, Clark MR, Stark L (1975) Dynamic overshoot in saccadic eye movements is caused by neurological control signed reversals. Exp Neurol 48:107-122.

Barash S, Melikyan A, Sivakov A, Zhang M, Glickstein M, Thier P (1999) Saccadic dysmetria and adaptation after lesions of the cerebellar cortex. J Neurosci 19:10931-10939.

Bloedel JR, Ebner TJ (1984) Rhythmic discharge of climbing fibre afferents in response to natural peripheral stimuli in the cat. J Physiol (Lond) 352:129-146.

Catz N, Dicke PW, Thier P (2005) Cerebellar complex spike firing is suitable to induce as well as to stabilize motor learning. Curr Biol 15:2179-2189.

Dean P, Mayhew E, Langdon P (1994) Learning and maintaining saccade accuracy: a model of brainstem-cerebellar interactions. J Cogn Neurosci 6:117-138.

Efron B, Tibshirani R (1993) An introduction to the bootstrap. New York: Chapman and Hall.

Fuchs AF, Robinson DA (1966) A method for measuring horizontal and vertical eye movement chronically in the monkey. J Appl Physiol 21:1068-1070.

Fuchs AF, Robinson FR, Straube J (1993) Role of the caudal fastigial nucleus in saccade generation. I. Neuronal discharge patterns. J Neurophysiol 10:1723-1740.

Gilbert PF, Thach WT (1977) Purkinje cell activity during motor learning. Brain Res 128:309-328.

Ito M (2005) Bases and implications of learning in the cerebellum: adaptive control and internal model mechanism. Prog Brain Res 148:95-109.

Kahlon M, Lisberger SG (2000) Changes in the responses of Purkinje cells in the floccular complex of monkeys after motor learning in smooth pursuit eye movements. J Neurophysiol 84:2945-2960.

Kitazawa S, Kimura T, Yin P-B (1998) Cerebellar complex spikes encode both destinations and errors in arm movements. Nature 392:494-497.

Kobayashi Y, Kawano K, Takemura A, Inoue Y, Kitima T, Gomi H, Kawato M (1998) temporal firing patterns of Purkinje cells in the cerebellar ventral paraflocculus during ocular following responses in monkeys. J Neurophysiol 80:832-848.

Kojima Y, Iwamoto Y, Yoshida K (2004) Memory of learning facilitates saccadic adaptation in the monkey. J Neurosci 24:7531-7539.

Marr D (1969) A theory of cerebellar cortex. J Physiol (Lond) 202:437-470.

Mays LE, Sparks DL (1980) Dissociation of visual and saccade-related responses in superior colliculus neurons. J Neurophysiol 43:207-232.

McLaughlin SC (1967) Parametric adjustment in saccadic eye movements. Percept Psychophys 2:359-362.

Miles FA, Lisberger SG (1981) Plasticity in the vestibulo-ocular reflex: a new hypothesis. Annu Rev Neurosci 4:273-299.

Munoz DP, Broughton JR, Goldring JE, Armstrong IT (1998) Age-related performance of human subjects on saccadic eye movement tasks. Exp Brain Res 121:392-400.

Noda H, Fujikado T (1987) Topography of the oculomotor area of the cerebellar vermis in macaques as determined by microstimulation. J Neurophysiol 58:359-378.

Ohtsuka K, Noda H (1991) Saccadic burst neurons in the oculomotor region of fastigial nucleus of the macaque monkeys. J Neurophysiol 65:1422-1434.

Ohtsuka K, Noda H (1995) Discharge properties of Purkinje cells in the oculomotor vermis during visually guided saccades in the macaque monkey. J Neurophysiol 74:1828-1840.

Ojakangas CL, Ebner TJ (1992) Purkinje cell complex and simple spike 
changes during a voluntary arm movement learning task in the monkey. J Neurophysiol 68:2222-2236.

Optican LM, Robinson DA (1980) Cerebellar-dependent adaptive control of primate saccadic system. J Neurophysiol 44:1058-1076.

Raymond JL, Lisberger SG (1998) Neural learning rules for the vestibuloocular reflex. J Neurosci 18:9112-9129.

Robinson FR, Fuchs AF, Noto CT (2002) Cerebellar influences on saccade plasticity. Ann NY Acad Sci 956:155-163.

Robinson FR, Noto CT, Bevans SE (2003) Effect of visual error size on saccade adaptation in monkey. J Neurophysiol 90:1235-1244.

Scudder CA, Kaneko CRS, Fuchs AF (2002) The brainstem burst generator for saccadic eye movements: a modern synthesis. Exp Brain Res 142:439-462.

Simmons DM (1979) Egg yolk embedding for frozen sections of whole primate brain segments. J Histotechnol 2:62-64.

Soetedjo R, Fuchs AF (2003) Climbing fiber discharges in the cerebellar oculomotor vermis change during saccadic adaptation. Soc Neurosci Abstr 29:274.11.

Soetedjo R, Kaneko CR, Fuchs AF (2002) Evidence that the superior colliculus participates in the feedback control of saccadic eye movements. J Neurophysiol 87:679-695.

Stone LS, Lisberger SG (1990) Visual responses of Purkinje cells in the cerebellar flocculus during smooth-pursuit eye movements in monkeys. II. Complex spikes. J Neurophysiol 63:1262-1275.

Straube A, Fuchs AF, Usher S, Robinson FR (1997) Characteristic of saccadic gain adaptation in rhesus macaques. J Neurophysiol 77:874-895.

Warabi T, Kase M, Kato T (1984) Effect of aging on the accuracy of visually guided saccadic eye movement. Ann Neurol 16:449-454.

Watanabe E (1984) Neuronal events correlated with long-term adaptation of the horizontal vestibulo-ocular reflex in the primate flocculus. Brain Res 297:169-174. 